University of South Florida

DIGITAL COMMONS

Digital Commons @ University of

@ UNIVERSITY OF SOUTH FLORIDA

South Florida

\title{
Effect of a preoperative warming intervention on the acute phase response of surgical stress
}

\author{
Vanda Doreen Wagner
}

University of South Florida

Follow this and additional works at: https://digitalcommons.usf.edu/etd

Part of the American Studies Commons

\section{Scholar Commons Citation}

Wagner, Vanda Doreen, "Effect of a preoperative warming intervention on the acute phase response of surgical stress" (2007). USF Tampa Graduate Theses and Dissertations.

https://digitalcommons.usf.edu/etd/2399

This Dissertation is brought to you for free and open access by the USF Graduate Theses and Dissertations at Digital Commons @ University of South Florida. It has been accepted for inclusion in USF Tampa Graduate Theses and Dissertations by an authorized administrator of Digital Commons @ University of South Florida. For more information, please contact digitalcommons@usf.edu. 
Effect of a Preoperative Warming Intervention on the

Acute Phase Response of Surgical Stress

by

\title{
Vanda Doreen Wagner
}

A dissertation submitted in partial fulfillment

of the requirements for the degree of

Doctor of Philosophy

College of Nursing

University of South Florida

\author{
Major Professor: Maureen Groër, R.N., Ph.D., FAAN \\ Jason Beckstead, Ph.D. \\ Clyde Gwinn, M.D. \\ Barbara Holtzclaw, Ph.D., R.N., FAAN
}

Date of Approval:

October 31, 2007

Keywords: hypothermia, forced-air warming, catecholamine, cytokine, innate immunity

(C) Copyright 2007, Vanda Doreen Wagner 


\section{Dedication}

I dedicate this dissertation to Brent and Karla with all my love.

You made this worthwhile journey so much easier. 


\section{Acknowledgements}

I would not have been able to complete this mind-expanding adventure without the guidance and support of many people. First and foremost, I want to express my heartfelt gratitude to Dr. Maureen Groër. As my dissertation chairperson, she provided me with unlimited support, guidance, and encouragement. Dr. Groër has been my mentor and has taught me what it takes to be a nurse scientist. My career as a nurse researcher will only benefit from her gracious gifts of knowledge.

Secondly, I would like to individually thank my committee members for their support and assistance in making this dissertation a reality. I would like to especially thank Dr. Jason Beckstead for his patience and direction in my search for understanding statistics. Heartfelt appreciation for his encouragement and feedback goes to Dr. Clyde Gwinn. I would also like to recognize and thank Dr. Barbara Holtzclaw for sharing her thermoregulation expertise with me. She will always be the "Temperature Lady!" Special thanks are given to Dr. Nagwa Dajani for being moderating Chairperson at my defense.

I also want to thank Dr. Mary Evans for her advisement and unfailing support. Warm thanks go to Alison Jones Montpetit and Marianne Chanti-Ketterl for the many hours of assistance, crises intervention, and friendship they gave so freely.

Last, but not least, I am very happy to acknowledge the financial funding I received from the Association of periOperative Registered Nurses and from the Linda Moody Doctoral Dissertation Scholarship of USF. This monetary support allowed me to reach for higher goals in my research efforts. 
Table of Contents

List of Tables $\quad$ iv

List of Figures $\quad$ V

Abstract vi vi

Chapter One: Introduction 1

Statement of the Problem 3

Focus of Inquiry 3

Definition of Terms 4

$\begin{array}{ll}\text { Purpose } & 8\end{array}$

Specific Aim $\quad 8$

$\begin{array}{ll}\text { Significance } & 8\end{array}$

$\begin{array}{ll}\text { Summary } & 9\end{array}$

Chapter Two: Context of the Study 11

$\begin{array}{ll}\text { Review of the Literature } & 11\end{array}$

$\begin{array}{ll}\text { Search Strategy } & 11\end{array}$

Physiology of Temperature Regulation 12

Etiology of Perioperative Hypothermia 14

$\begin{array}{ll}\text { Immune Responses to Surgery } & 16\end{array}$

Immune Responses to Hypothermia 19

Therapeutic Effects of Prewarming 23

$\begin{array}{ll}\text { Discussion } & 26\end{array}$

$\begin{array}{ll}\text { Conceptual Model } & 27\end{array}$

$\begin{array}{ll}\text { Study Hypotheses } & 29\end{array}$

$\begin{array}{ll}\text { Summary } & 30\end{array}$ 
Chapter Three: Method 31

Research Design 31

Protection of Human Participants $\quad 32$

Study Approval $\quad 32$

Recruitment Process 32

Informed Consent Process 32

Benefits and Risks of Participation $\quad 32$

Sample Selection 34

Study Setting 35

Inclusion and Exclusion Criteria $\quad 35$

Data Collection 36

Intervention Protocol 37

$\begin{array}{ll}\text { Serum Collection Protocol } & 37\end{array}$

Assay Protocol 38

Data and Safety Monitoring $\quad 40$

Data Analysis $\quad 41$

$\begin{array}{ll}\text { Summary } & 42\end{array}$

Chapter Four: Results 43

Data Preparation 43

Profile of Study Sample $\quad 44$

$\begin{array}{ll}\text { Statistical Analyses } & 46\end{array}$

Test Assumptions $\quad 47$

Hypotheses, Statistical Procedures, and Results $\quad 47$

Hypothesis $1 \quad 47$

Hypothesis $2+49$

Cortisol 50

Epinephrine $\quad 51$

Norepinephrine 53

Hypothesis 3

$\begin{array}{ll}\text { IL-1 } \beta & 55\end{array}$ 
IL-6 56

$\begin{array}{ll}\text { TNF- } \alpha & 58\end{array}$

$\begin{array}{ll}\mathrm{IFN}-\gamma & 60\end{array}$

$\begin{array}{ll}\text { CRP } & 61\end{array}$

Summary $\quad 63$

Chapter Five: Discussion $\quad 64$

Specific Aim of the Study $\quad 64$

Hypothesis $1 \quad 65$

$\begin{array}{ll}\text { Hypothesis } 2 & 68\end{array}$

Cortisol $\quad 69$

Catecholamines $\quad 70$

Hypothesis $3 \quad 72$

$\begin{array}{ll}\text { Study Limitations } & 74\end{array}$

Implications for Nursing $\quad 75$

Implications for Future Research $\quad 76$

$\begin{array}{ll}\text { Summary } & 77\end{array}$

$\begin{array}{ll}\text { References } & 78\end{array}$

$\begin{array}{lr}\text { Appendices } & 88\end{array}$

Appendix A: IRB Approval $\quad 89$

Appendix B: Recruitment Flyer $\quad 91$

Appendix C: Informed Consent Form 92

Appendix D: Surgeon Letter 101

Appendix E: Anesthesia Provider Letter 102

Appendix F: Data Collection Forms 103

$\begin{array}{ll}\text { About the Author } & \text { End Page }\end{array}$ 


\section{List of Tables}

Table 1 Descriptive Statistics Calculations on Temperature Across Time 44

Table 2 Participant Characteristics 45

Table 3 Descriptive Statistics of Temperature $\left({ }^{\circ} \mathrm{F}\right)$ Across Time 49

Table 4 Raw Mean Values of Cortisol (ng/ml) Across Time 50

Table 5 Raw Mean Values of Epinephrine (pg/ml) Across Time 52

Table 6 Raw Mean Values of Norepinephrine (pg/ml) Across Time 54

Table 7 Raw Mean Values of IL-1 $\beta$ (pg/ml) Across Time 55

Table 8 Raw Mean Values of IL-6 (pg/ml) Across Time 57

Table 9 Correlation Coefficients for IL-6 and TNF- $\alpha$ Across Time 57

Table 10 Raw Mean Values of TNF- $\alpha$ (pg/ml) Across Time 59

Table 11 Raw Mean Values of IFN- $\gamma(\mathrm{pg} / \mathrm{ml})$ Across Time $\quad 60$

Table 12 Raw Mean Values of CRP (pg/ml) Across Time 62

Table 13 Correlations Between BMI and CRP Across Time 63

Table 14 Total Mean Temperature $\left({ }^{\circ} \mathrm{F}\right)$ of Hypothermic Participants at Time $3 \quad 66$

Table 15 Total Mean Temperature ( $\left.{ }^{\circ} \mathrm{F}\right)$ of Hypothermic Participants at Time $4 \quad 66$ 


\section{List of Figures}

$\begin{array}{lll}\text { Figure } 1 \text { Conceptual Model } & 28\end{array}$

Figure 2 Flow Diagram of Participant Status During the Study 34

Figure 3 Mean Temperature Values ( $\left.{ }^{\circ} \mathrm{F}\right)$ Across Time 48

Figure 4 Raw Mean Values of Cortisol (ng/ml) Across Time 51

Figure 5 Raw Mean Values of Epinephrine (pg/ml) Across Time 53

Figure 6 Raw Mean Values of Norepinephrine (pg/ml) Activity Across Time 54

Figure 7 Raw Mean Values of IL-1 $\beta$ (pg/ml) Across Time 56

Figure 8 Raw Mean Values of IL-6 (pg/ml) Across Time 58

Figure 9 Raw Mean Values of TNF- $\alpha$ (pg/ml) Activity Across Time 59

Figure 10 Raw Mean Values of IFN- $\gamma(\mathrm{pg} / \mathrm{ml})$ Across Time 61

Figure 11 Raw Mean Values of CRP (pg/ml) Across Time 62 
Effect of a Preoperative Warming Intervention on the Acute Phase

Response of Surgical Stress

Vanda Doreen Wagner

\begin{abstract}
When a patient is exposed surgical stress, the endocrine system secretes hormones in response to that stress. These hormones further activate the immune system to release cytokines and other acute phase reactions. These processes are supposed to protect the body by upregulating the innate immune system and producing an inflammatory response that acts to protect and heal. However, uncontrolled surgical stress may cause a weaker immune response that may lead to delayed wound healing.

The phenomenon of unplanned perioperative hypothermia is known to expose patients to additional surgical stress. The purpose of this preliminary experimental study was to determine the effect of a preoperative warming intervention on the acute phase response of surgical stress in surgical patients. Specifically, the aim of this study was to evaluate the effect of a prewarming intervention using a forced-air warming (FAW) device versus routine care (RC) using warmed cotton blankets on the development of unplanned hypothermia, cytokine production, and endocrine responses. It was hypothesized that 1) the FAW participants would experience less unplanned perioperative hypothermia than the RC participants; 2) the FAW participants would experience lower catecholamine and cortisol levels than the RC participants; and 3) the FAW participants
\end{abstract}


would experience higher proinflammatory cytokine and CRP production intra- and postoperatively than the RC participants.

Infrared tympanic temperatures and 4 blood samples were taken at 4 time intervals from each of the $28(n=14$ each group) randomized participants that underwent routine general anesthesia surgery. Serum concentrations of CRP, cortisol and IL-1 $\beta$, IL6 , TNF- $\alpha$, and IFN- $\gamma$, and plasma concentrations of epinephrine and norepinephrine were measured. To test the hypotheses across time and between groups, a repeated measures ANOVA design was used.

Though FAW was not associated with a differential endocrine or inflammatory response in this small, preliminary study, further study of forced air warming as a preoperative nursing intervention is warranted. The finding of higher than expected IL-6 levels in the preoperative period suggests a potential role for anxiety, an important factor in psychoneuroimmunological pathways, that could affect recovery and healing. The relationship between surgical stress, anxiety, and preoperative IL-6 deserves further study. 


\section{Chapter One: Introduction}

Perioperative patients commonly dominate the care provided by hospitals and freestanding facilities in the United States as shown by Vital Health Statistics from the Centers for Disease Control and Prevention (CDC). According to summary findings for 1996, more than 40 million surgical procedures were performed for hospitalized patients with $30 \%$ of those surgeries considered elective procedures. In both hospitals and freestanding surgical centers, another 31.5 million patients had ambulatory surgery that year (CDC, 1998). Because of cost containment measures and technological advances, patients are sent home earlier or considered for ambulatory surgery that leads to discharge within hours of surgery completion. However, even with an earlier discharge home, patients still have to recover for weeks to several months after a surgical procedure because of the impact that surgical stress has on the body systems.

An increased understanding of how surgical stress affects patients has occurred in the last 70 years with much evidence indicating that physiologic stress reduction decreases complications and improves perioperative outcomes (Kehlet \& Wilmore, 2002; Wilmore, 2002). In the last decade alone, preventive therapies recognized to reduce complications have led to changes in practice and standardized perioperative strategies, such as minimally invasive procedures, timing of preoperative antibiotics, perioperative insulin therapy, and postoperative thromboembolic prophylaxis (Kehlet \& Wilmore, 2002; Meiler, 2006). 
Surgery is a threatening experience because of multiple stressful components. Stress is a term used to refer to "forces or factors that cause disequilibrium to an organism and therefore threaten homeostasis" (Wilmore, 2002). Surgical stress results from cellular and organ system insult, tissue injury, and nociceptive stimulation that impact endocrine and metabolic processes. The central nervous system, hypothalamicpituitary-adrenal axis, and the peripheral autonomic nervous system are all involved in an integrated response, known collectively as the stress response, in order to return the patient to homeostasis following surgery.

When a patient is exposed to surgical stress, the endocrine system secretes various hormones, such as cortisol and catecholamines, in a response mediated by the hypothalamic-pituitary-adrenal (HPA) axis and the autonomic nervous system. It is well established that both external and internal stressors, such as preoperative anxiety, tissue injury, and anesthesia medications, associated with the surgical experience activate the HPA axis and that the HPA axis plays a key role in stress responses (Haddad, Saade, \& Safieh-Garabedian, 2002). Cytokines, such as interleukin-1 beta (IL-1 13 ), interleukin-6 (IL-6), and tumor necrosis factor-alpha (TNF- $\alpha$ ), are released in response to signals produced by surgical tissue damage, anesthesia, sympathetic activation, and psychosocial stress. IL-6 in particular is a multifunctional cytokine that is the major stimulator of acute phase reactants, including $\mathrm{C}$-reactive protein (CRP) and other hepatic proteins (Ogawa et al., 2000). This complicated response is intended to protect the body by producing an inflammatory state that acts to protect and heal. Any continued stimulation of the stress response, however, may lead to reduced cardiac function, a diminished immune response to infection, delayed wound healing, and a general loss of energy stores necessary to 
promote healing (Edwards, 2003). An imbalance between inflammatory and antiinflammatory cytokines causes immunosuppression. Restoration of an adequate balance between inflammatory and anti-inflammatory immune cell function is the key to decreasing surgical stress.

With recognition that perioperative nursing practices could prevent later complications comes the need to reassess existing practices and how those practices may or may not impact patient risks. To provide best practice, it is sometimes necessary to question traditional interventions in order to look for new approaches to reduce surgical stress. For example, unplanned perioperative hypothermia is still accepted as commonplace and even just expected. The traditional practice is to treat perioperative hypothermia, rather than preventing its development. It is well established that unplanned hypothermia contributes to the stress response and increases the risk for postoperative infections (Beilin et al., 1998; Kurz, Sessler, \& Lenhardt, 1996). Since all surgical patients are potentially at risk for developing hypothermia, they are routinely put at risk for developing surgical stress induced complications such as infection.

\section{Statement of the Problem}

\section{Focus of Inquiry}

In order to study the reduction of surgical stress related to unplanned perioperative hypothermia, a focused inquiry into how a nursing intervention would impact the initial phase of surgical stress was selected. The specific intervention used in this focused study was a preoperative forced-air warming intervention.

Perioperative hypothermia, a core body temperature less than $96.8^{\circ} \mathrm{F}\left(36^{\circ} \mathrm{C}\right)$, happens during surgical procedures in two ways: planned or unplanned (AORN, 2007; 
Sessler, 1997a, 1997b). Planned hypothermia is used during surgeries that benefit from a lessened metabolic demand and organ protection during low blood flow periods, such as cardiac and neurological surgery. Conversely, unplanned hypothermia occurs because of the inherent heat loss in the operating room (OR), of anesthesia-induced thermoregulation impairment, and the actual surgery itself. Other factors that place patients at risk for unplanned hypothermia include major fluid or blood loss, infusion of cold fluids/blood, large volume of non-warmed irrigation, exposure of a large body cavity, patient's age (pediatric or geriatric specifically), and the patient's physical status (Forstot, 1995; Sessler, 2000). Unplanned hypothermia is considered one of the most common and expected complications that patients experience during surgery.

There is a large amount of literature addressing the numerous complications that occur because of unplanned perioperative hypothermia (Beilin et al., 1998; Connor \& Wren, 2000; Guest, Vanni, \& Silbert, 2004; Holtzclaw, 1997; Kurz, Sessler, \& Lenhardt, 1996; Lee, Battistella, \& Go, 2001; Lenhardt et al., 1997; Leslie \& Sessler, 2003; Schein, Rucinski, \& Wise, 1996). However, there is little research focusing on the prevention of unplanned perioperative hypothermia as a preoperative nursing intervention. Therefore, a preliminary investigation of preoperative warming as a nursing intervention to reduce surgical stress was conducted.

\section{Definition of Terms}

The definitions listed below provide the reader with a lexicon that will be useful in reading this dissertation. These terms are used frequently throughout this document and are standard definitions in the field. 
Acute Phase Proteins (APPs)

Proteins whose plasma concentration increases or decreases by at least $25 \%$ in order to defend the host during inflammatory processes. Cytokines are the primary stimulators of the liver to produce the APPs.

Acute Phase Response (APR)

The metabolic, physiologic, and behavioral changes that accompany the organism's response to inflammation and infection. The major cell involved in generating the APR is the macrophage.

Active Warming

The application of conductive, convective or radiative warming devices to clinically increase the body temperature.

\section{Catecholamines}

A group of aromatic amines (norepinephrine, epinephrine, dopamine, and their derivatives) that act as hormones and neurotransmitters.

Epinephrine. An amine that functions as both a neurotransmitter in the central nervous system (CNS) and a hormone when released from the adrenal medulla. It mobilizes the body for action.

Norepinephrine. An amine released by both the adrenal medulla and neurons in the CNS and the sympathetic nervous system (SNS), which induces arousal. C-Reactive Protein (CRP)

An alpha globulin synthesized in the liver and normally present in trace amounts in circulating blood. CRP is one of the acute-phase proteins, levels of which rise during 
responses to varied stimuli, such as rheumatoid arthritis, inflammatory diseases, and surgical trauma. It is used as an indicator of the inflammatory process.

\section{Core Temperature}

The temperature in the thermal compartment of the body that is composed of highly perfused tissues and major organs where the temperature is uniform and high compared with the rest of the body.

\section{Cortisol}

The main corticosteroid secreted in humans by the adrenal cortex with the main biological effects of gluconeogenesis, increased glucose concentration, fat metabolism, anti-inflammatory, and anti-immune actions.

\section{Cytokines}

Proteins that are classified into interferons, lymphokines, monokines, and chemokines that influence the activity of other cells by intercellular signals. Cytokines, as a group, are responsible for the multidirectional communication among cells engaged in host defense, tissue repair and other immune responses.

$I L-1 \beta$. Pro-inflammatory monokine that plays a major role in the induction of acute phase reactant synthesis. Stress induced IL-1 $\beta$ activation of the HPA axis is modulated by IL-6.

IL-6. Pro-inflammatory lymphokine that has a role in stress-induced activation of the HPA axis. Its main function is to stimulate the production of most acute phase proteins and potentiate the effects of other cytokines

$I F N-\gamma$. Proinflammatory interferon that amplifies acute phase response. Secretion of IFN $\gamma$ is a hallmark of Th1 lymphocytes. 
$T N F-\alpha$. A proinflammatory paracrine that has a major role in mediation of the inflammatory response and the induction of acute phase proteins.

Forced-air warming (FAW)

Convection warming technology that disperses a "blanket" of warm air over the patient's skin in a controlled manner.

\section{Hypothermia}

A core temperature less than $96.8^{\circ} \mathrm{F}\left(36^{\circ} \mathrm{C}\right)$.

\section{Normothermia}

A core temperature range of $96.8^{\circ} \mathrm{F}\left(36^{\circ} \mathrm{C}\right)$ to $100.4^{\circ} \mathrm{F}\left(38^{\circ} \mathrm{C}\right)$.

Passive warming

Insulation type warming approaches, such as warmed cotton blankets, socks, and head coverings, used to insulate the body from heat loss through radiation.

\section{Redistribution Hypothermia}

A decrease in body temperature occurring as heat is exchanged from the body's core compartment to the peripheral tissues.

Stress

A physiologic response from physical or psychological factors that cause disequilibrium to an organism and therefore threatens homeostasis. Surgical Stress

An invasive action that causes cellular and organ system insult, tissue injury, and nociceptive stimulation that impacts endocrine and metabolic processes.

\section{Thermoregulation}

The action of monitoring and regulating body temperature. 


\section{Purpose}

The overall objective of this preliminary experimental study was to determine the effect of a preoperative warming intervention on the initial acute phase response of surgical stress in perioperative patients. The use of either a FAW or routine practice of preoperative warming intervention served as the independent variable. The effects of this manipulation on thermoregulation and acute phase stress response were examined. Specifically, three questions were addressed: 1) What is the effect of type of preoperative warming on the occurrence of unplanned perioperative hypothermia? 2) What is the effect of type of preoperative warming on the acute phase response of surgical stress during the perioperative period (i.e., changes in cortisol, CRP, IL-1ß, IL-6, IFN- $\gamma$, TNF- $\alpha$, epinephrine and norepinephrine)? and 3) Is there a difference in the level of surgical stress response between the FAW group of patients versus the routine care (RC) group of participants?

Specific Aim

The overreaching objective of the study was to obtain new information about a nursing intervention that could possibly decrease surgical stress. The specific aim of this preliminary study was to examine the effect of a preoperative warming intervention using a FAW device versus routine care using warmed cotton blankets on the development of unplanned perioperative hypothermia, pro-inflammatory cytokine production, and neuroendocrine responses.

\section{Significance}

Perioperative nurses provide patient care during the preoperative, intraoperative, and postoperative phases of a surgical procedure. Throughout these phases of care, the 
professional nurse takes on the vital role of patient advocate with an emphasis on the maintenance of a patient's psychological and physical well being. Nurses are educationally prepared to provide organized patient care planned on an individualized basis and encompassing the biophysiological, psychological, and social components of the patient's response or adjustment to physical alterations. Thus, perioperative nursing is a specialized area of practice that provides continuity of care and treatment necessary to provide comfort to the patient, to assist the patient in promotion and maintenance of health, and the prevention, detection, and treatment of illness.

The significance of this research was in the development of a new understanding of surgical stress and how the perioperative nurse may impact and perhaps reduce surgical stress. The focus of this study created opportunities to learn more about the phenomenon of redistribution hypothermia and surgical stress. This study evaluated the effect of a FAW intervention that was hypothesized to lead to less perioperative hypothermia, lower levels of stress hormone production, a heightened inflammatory response and less immunosuppression, that would ultimately impact overall surgical stress of the perioperative patient. The knowledge gained from the benefits of a preventive nursing intervention could potentially provide evidence and direction for best practices during the entire perioperative experience.

\section{Summary}

Perioperative nurses care for patients before, during and after surgery. The known problem of unplanned hypothermia adds to the surgical stress that all patients experience. Numerous studies have provided an increased understanding of how surgical stress affects patients and that prevention measures can be used to reduce perioperative 
complications. A lack of specific evidence related to the prevention of unplanned perioperative hypothermia and how it may be a possible surgical stress reduction strategy was recognized and the need for a nursing intervention study was determined. A preliminary investigation of preoperative FAW intervention and its effect on surgical stress was conducted to answer the posed research questions. The following chapters provide a discussion about the context of the study, the research method, results of the study, and an in depth discussion of the study findings. 


\section{Chapter Two: Context Of Study}

In this chapter, the review of literature and conceptual framework underlying the study is discussed. A brief review of the strategies undertaken for literature search is followed by a discussion of specific literature pertaining to the physiology of temperature regulation, etiology of perioperative hypothermia, cellular immune responses to hypothermia, immune responses to surgery, and the therapeutic effects of prewarming. The evidence provided support that prevention of unplanned hypothermia could be used as an effective nursing strategy to significantly reduce the stress of surgery and improve patient outcomes. The conceptual model used to provide the framework for this research is discussed prior to the outline of the research questions and study hypotheses.

\section{Review of Literature}

\section{Search Strategy}

Several literature search strategies were used. The standard online searches with Medline, Pubmed, and Biomed Central came up with over 20,000 hits for the key words perioperative hypothermia. The author found surgical hypothermia studies being conducted in the 1950's and that animal studies prompted the questions still studied today. Further search for terms acute phase reaction and surgery delivered 56 hits and surgical stress alone came up with 724 hits. With limits placed to English language only and the elimination of pediatric, dental, obstetric and cardiac surgeries, and exercise physiology, a reasonable retrieval of literature occurred. Further review of reference sections and bibliographies of all retrieved articles were examined for additional studies. 
After reviewing articles for specificity to perioperative hypothermia and surgical stress, the literature search finally yielded 156 articles for intense review.

Physiology of Temperature Regulation

The human body temperature is a tightly controlled physiological parameter that is normally controlled within $0.36^{\circ} \mathrm{F}\left(0.2^{\circ} \mathrm{C}\right)$. Even small variations in the core body temperature incite aggressive thermoregulatory defenses, because maintenance of body temperature is necessary for life. The precision of thermoregulatory control is similar in men and women, but declines in the elderly (Lopez, Sessler, Walter, Emerick, \& Ozaki, 1994; Vassilieff, Rosencher, Sessler, \& Conseiller, 1995).

The three major autonomic thermoregulatory defenses in humans are vasoconstriction, shivering, and sweating (Sessler \& Akca, 2002). Each of these defenses has a threshold (triggering core temperature), a gain (incremental change in response), and a maximum intensity response (Sessler, 1997b). Sweating and vasoconstriction thresholds are separated only by a few tenths of a degree centigrade, whereas, the shivering threshold is a full degree centigrade below the vasoconstriction threshold. This inter-threshold range defines the normal range of body temperature and is based on the individual's "set point" which ranges from approximately 97.5 to $99.14^{\circ} \mathrm{F}$ (36.4 to 37. $3^{\circ} \mathrm{C}$ ) (Holtzclaw, 1993; Sessler, 1997b).

The thermoregulatory system consists of a sensory component, a control center, and effector mechanisms. The control center, situated in the hypothalamus, maintains normothermia by balancing heat production, heat conservation, and heat loss. The hypothalamus regulates temperature hormonally. Peripheral thermoreceptors in the skin and central thermoreceptors in the hypothalamus, spinal cord, abdominal organs, and 
other central locations provide the hypothalamus with information about skin and core temperatures (Buggy \& Crossley, 2000; Sessler, 1997b, 2000).

If skin and core temperatures are low, the hypothalamus responds by triggering heat production and heat conservation mechanisms. Increased heat production is initiated by a series of hormonal mechanisms involving the hypothalamus and its connections with the endocrine system. The heat producing mechanism begins with a hypothalamic hormone, thyrotropin-stimulating hormone releasing hormone (TSH-RH). TSH-RH in turn stimulates the anterior pituitary to release thyroid-stimulating hormone (TSH), which acts on the thyroid gland, stimulating release of thyroxine (T4), one of the thyroid hormones. This hormone then acts on the adrenal medulla, causing the release of epinephrine (a catecholamine and vasopressive hormone) into the bloodstream (Silva, 2005). Epinephrine causes vasoconstriction, stimulates glycolysis, and increases metabolic rates, thus increasing heat production (Buggy \& Crossley, 2000; Sessler, 1997b, 2000). Other hormonal changes influence temperatures, such as ovulatory cycles of women and the diurnal variations that occur in all people. Plasma cortisol concentrations are thought to influence the 24-hour circadian variations in human temperatures by $0.2^{\circ}$ to $0.3^{\circ} \mathrm{C}$ with temperatures falling during sleep and peaking in the late afternoon (Holtzclaw, 1993).

The hypothalamus also triggers heat conservation. The mechanisms of heat conservation involve stimulating the sympathetic nervous system, which is responsible for stimulating the adrenal cortex, increasing skeletal muscle tone, initiating the shivering response, and producing vasoconstriction. The hypothalamus also functions in raising body temperatures by relaying information to the cerebral cortex. Awareness of cold 
provokes voluntary responses such as increased body movement (Buggy \& Crossley, 2000; Holtzclaw, 1993; Sessler, 1997b, 2000).

\section{Etiology of Perioperative Hypothermia}

Unplanned perioperative hypothermia is a common and potentially preventable occurrence in surgical patients. Hypothermia is one of the most common complications that surgical patients experience (Sessler, 1997b). Numerous clinical trials have demonstrated that unplanned hypothermia increases the incidence of serious complications including surgical site infections, (Flores-Maldonado, Medina-Escobedo, Rios-Rodriguez, \& Fernandez-Dominguez, 2001; Kurz, Sessler, \& Lenhardt, 1996) adverse cardiac events, (Frank et al., 1993) increased blood loss, (Schmied, Kurz, Sessler, Kozek, \& Reiter, 1996) altered drug metabolism, (Heier et al., 2002) and extended postanesthesia recovery time (Lenhardt et al., 1997).

When a surgical patient undergoes general anesthesia for periods longer than one hour, hypothermia is expected because anesthesia disrupts the behavioral and physiological mechanisms of thermoregulation (Buggy \& Crossley, 2000; Sessler \& Akca, 2002). During that first hour of anesthesia, the patient's core temperature usually drops $0.9^{\circ} \mathrm{F}$ to $2.7^{\circ} \mathrm{F}\left(0.5^{\circ} \mathrm{C}\right.$ to $\left.1.5^{\circ} \mathrm{C}\right)$ (Matsukawa et al., 1995). This drop in core temperature can be explained by redistribution of heat from the body's core to periphery. Redistribution occurs because anesthetics inhibit thermoregulatory control and therefore disrupt the tonic vasoconstriction that normally maintains a core-to-peripheral temperature gradient. This redistribution is not a clear exchange of heat with the environment, but a heat flow from the actual core to the periphery, thereby reducing core temperature (Matsukawa et al., 1995; Sessler \& Akca, 2002). 
The next phase of the hypothermic action is a slower, linear decrease in core temperature. Simply, it results from heat loss exceeding heat production. This phase lasts approximately 2-3 hours and depends on the difference between heat loss and metabolic heat production. Approximately $90 \%$ of heat loss is through the skin surface, with convection and radiation usually contributing more to the process than evaporation or conduction (Kurz, Sessler, Christensen, \& Dechert, 1995; Sessler, 2000),

After 3 to 5 hours of anesthesia, there is a plateau phase in hypothermic patients. This may reflect a steady state of heat loss equaling heat production and seen in patients who are well insulated. However, if a patient is quite hypothermic, the halt to the temperature decline results from activation of thermoregulatory vasoconstriction, which decreases cutaneous heat loss and acts to hold metabolic heat in the body core. Characteristically, this happens when the patient's core temperature is around $93.2^{\circ} \mathrm{F}$ $\left(34^{\circ} \mathrm{C}\right)$ (Kurz, Sessler, Christensen, \& Dechert, 1995).

The risk of unplanned hypothermia is greater in certain patients, such as neonates, trauma patients, and patients with extensive burns (Caldwell, Wallace, \& Cone, 1994; Gentilello, Jurkovich, Stark, Hassantash, \& O'Keefe, 1997; Macario \& Dexter, 2002; Rutherford et al., 1998; Tander et al., 2005). Other factors that place patients at risk for unplanned hypothermia development include major fluid loss, infusion of cold fluids, exposure of a large body cavity, increased duration of anesthesia time, and the patient's co-morbidity status (Forstot, 1995; Sessler, 1993). 


\section{Immune Responses to Surgery}

Cytokines mediate and regulate immune and inflammatory responses. The complex interaction that exists between cytokines, inflammation and the adaptive response in maintaining homeostasis is crucial to survival just like it is in the stress response. Most studies examining immune responses to surgery focus on the intraoperative and postoperative phases of the surgical experience.

During inflammation, the initiation of the stress system, because of the effect of cortisol, protects the organism from systemic "overshooting" with proinflammatory cytokines (Elenkov, Iezzoni, Daly, Harris, \& Chrousos, 2005). Homeostasis relies on cytokines as they play vital roles in mediating inflammatory and immune responses. Cytokines may act in a paracrine, autocrine or endocrine manner in order to control the proliferation, differentiation and activity of immune cells (Elenkov, Iezzoni, Daly, Harris, \& Chrousos, 2005; Elenkov, Papanicolaou, Wilder, \& Chrousos, 1996).

In one of the first studies that demonstrated changes in cytokine serum levels after surgery related to the extent and duration of the surgical procedure and the type of disease process, Baxevanis and others (1994) studied 80 patients scheduled for elective surgery. Serum levels of IL-2, IL-1 $\beta$, IL-6, TNF- $\alpha$, and prostaglandin E2 (PGE2) were measured serially pre- and postoperatively to determine the surgical stress differences between surgery types and relative immune responses. The data suggested that overproduction of inflammatory cytokines in vivo together with a decrease of IL-2 levels were responsible for postoperative immunosuppression and opened the possibility of using immune specific interventions for the prevention of postoperative infections (Baxevanis, Papilas, Dedoussis, Pavlis, \& Papamichail, 1994) 
IL-6 is a T cell helper 2 (Th2) cytokine, and the main mediator of acute phase response and also has many biologic activities such as the stimulation of hepatocytes and the production of acute phase reactants such as CRP. The increase of IL-6 seen during tissue injury can be interpreted as being related to the predominance of Th2 cells caused by surgical stress (Ogawa et al., 2000). In a study of 20 gastrointestinal cancer patients age 44-66, Ogawa and others (2000) measured hormones, cytokines and acute phase reactants from the preoperative to postoperative time frames. The researchers found that during the perioperative period, catecholamines and cortisol were significantly increased during and after the operation. The serum IL-6 was increased after the surgery whereas CRP was decreased intraoperatively with only a postoperative increase. Th cells were decreased by surgical stress and the Th1 to Th2 balance was upset, with Th2 cells predominant due to the hypersecretion of cortisol (Ogawa et al., 2000).

Biffl and colleagues (1996) performed a review of literature in order to summarize origins, actions, and biologic effects of IL-6 as well as the IL-6 response to injury. Prolonged and excessive elevations of circulating IL-6 levels in patients after elective surgery have been associated with complications and even death. Findings included the role of IL-6 in the acute phase response to surgery and it was suggested that IL-6 is a more accurate indicator of inflammatory status than acute phase proteins, especially in patients with liver disease. Surgery provoked increases in IL-6 levels within 1 to 3 hours postoperatively which remained elevated for up to 72 hours in uncomplicated cases. It was also found that the magnitude of elevation was related directly to the degree of surgical insult. An interesting result of the review was the finding that IL-6 response to tissue injury in septic patients appeared to be independent of commonly summoned 
mediators, such as TNF, IL-1 $\beta$ and endotoxin. The most confirmatory finding in this review was that surgery causes a paradox. This paradox occurs when the hyperinflammatory state predisposes patients to the development of immunosuppression. When there is tissue insult, physiologic response involves an early hyperinflammatory response, which is complemented by a degree of compensatory anti-inflammatory effect. If this early inflammatory response is excessive, an inappropriate persistence of antiinflammatory compensation will result in immunosuppression (Biffl, Moore, Moore, \& Peterson, 1996).

The initiation of the inflammatory response and the cessation of this process during the perioperative experience are not well understood (Bjornsson et al., 2007). Bjornsson and colleagues (2007) studied the early phase of acute inflammatory reaction and the course of pro- and anti-inflammatory cytokines in relation to the adrenal response to surgical stress. They measured IL-1 $\beta$, IL-6, TNF- $\alpha$, IL-8, IL-12 and IL-10 in 13 patients ( $67 \pm 9$ years) undergoing total hip replacement due to osteoarthritis. The measurement of cortisol was interpreted to be related to the operative stress level of each patient. There was a significant inverse correlation between the cortisol secretion and IL6 response during the first 24 hours after surgery that demonstrated cortisol as having a slowing effect on the acute inflammatory response. Five patients had significantly higher cortisol levels preoperative than those 8 patients who had double their cortisol concentration postoperatively; $580 \pm 128 \mathrm{nmol} / 1$ versus $33 \pm 123 \mathrm{nmol} / 1(\mathrm{p}=.003)$. This led the researchers to question if IL-6 drove cortisol production or if cortisol could be a negative feedback loop in the inflammatory system (Bjornsson et al., 2007). 
In a study that examined CRP levels after different hip surgeries in 349 patients, researchers found CRP levels could be used to quantify the degree of tissue damage and invasiveness of a procedure. Peak CRP levels were reached on the second postoperative day in each group (median range of CRP levels $8.7-16 \mathrm{mg} / \mathrm{dL}$ ). Significant differences were found between each of the 5 types of hip surgeries and high CRP values on the second postoperative day was found to indicate a major inflammatory response to the surgery and reflected the level of tissue damage. The highest levels of mean CRP and resultant surgical stress were found in the hemiarthroplasty $(16 \mathrm{mg} / \mathrm{dL} ; \mathrm{p}=<0.001)$ and total hip arthroplasty patients $(16 \mathrm{mg} / \mathrm{dL} ; \mathrm{p}=<0.001)$ (Neumaier, Metak, \& Scherer, 2006).

Ruzic and colleagues (2004) studied the differences in systemic stress responses in 80 patients undergoing 3 different types of surgery for benign prostatic hyperplasia by measuring urinary cortisol, and CRP, and fibrinogen as well as antioxidants from serum samples. The researchers found significantly higher levels of cortisol, CRP and total antioxidant status in all three study groups after surgery. A strong systemic stress response intra- and postoperatively was related to the surgery and blood loss. The researchers also commented that cortisol could be increased by fear, smoking, hypovolemia and endocrine dysfunction (Ruzic et al., 2005).

Immune Responses To Hypothermia

A study by Beilin and others (1998) investigated the postoperative impact of mild intraoperative hypothermia in comparison to normothermia in terms of cellular immune responses. Sixty patients undergoing abdominal surgery (hysterectomy, colorectal surgery, and open cholecystectomy) were randomly assigned to either routine care $(\mathrm{n}=30)$ 
or FAW ( $\mathrm{n}=30)$. The routine care group (hypothermia group) did not receive any active warming nor warmed fluids during the perioperative period. The FAW group (normothermia group) received active warming that began 45 minutes before anesthesia induction and continued throughout the surgery as well as 1 hour postoperatively. The normothermia group also received warmed $\left(98.6^{\circ} \mathrm{F} / 37^{\circ} \mathrm{C}\right)$ intravenous (IV) fluids. Anesthesia care was standardized for all patients. Venous blood samples were collected 90 minutes before anesthesia induction, at the end of surgery, and at 24 and 48 hours postoperatively. Within 1 hour after the blood was drawn, PBMC were isolated and suspended in calf serum and then frozen until use within 1 month's time. After quick thaw, cells were washed and viability tested by trypan blue dye exclusion. Cells were used only if viability was $>95 \%$. PBMCs were used to test cytokine production IL-1, IL2, and IL-6, TNF $\alpha$, mitogen-induced proliferation and NK cell cytotoxicity. Plasma cortisol levels were also determined. Statistical analysis was done by repeated measures ANOVA with probability values $<0.05$ considered significant (Beilin et al., 1998).

Mean core temperature was significantly less in the hypothermia group throughout the surgery and until 3 hours after surgery. Mitogen induced proliferation assay results showed significant suppression with the hypothermic group that suggests suppressive effects involve various lymphocyte subpopulations. The mitogen proliferation in the normothermia group remained stable at all time periods. Production of IL-1 and IL-2 was significantly higher 24 hours after surgery in the normothermia group compared to the hypothermia group. The researchers state that because IL-2 plays such a central role in various immune responses, a reduction of this cytokine would impair immune defense mechanisms and increase susceptibility to surgical site infections (SSIs). 
The proinflammatory cytokine IL-1 increase noted in the normothermia group confirms other studies; and suggests that delayed wound healing found after hypothermia could be partially accounted for by reduced IL-1 production in hypothermic patients. No significant differences were found in NK cell cytotoxicity between the two groups at any repeated measurement. Lastly, there were significant elevations in the cortisol levels in the hypothermic group as compared to the normothermia group at 24 hours after surgery. The researchers conclude that these findings indicate perioperative hypothermia may contribute to the suppression of immune response in the perioperative period (Beilin et al., 1998).

Frank and colleagues (1995) examined the relationship between perioperative changes in body temperature and plasma levels of stress hormones in 74 elderly patients undergoing abdominal, thoracic, or lower extremity vascular surgical procedures. Randomly assigned to FAW $(\mathrm{n}=37)$ or routine care $(\mathrm{n}=37)$, the two groups had core temperatures, skin temperatures, and plasma concentrations of epinephrine, norepinephrine, and cortisol measured and compared throughout the perioperative period. In addition, heart rate and arterial blood pressure were compared between groups. The mean core temperature was lower in the routine care group on admission to the recovery area $\left(35.3^{\circ} \mathrm{C} \pm 0.1^{\circ} \mathrm{C}\right)$ compared to the FAW $\left(36.7^{\circ} \mathrm{C} \pm 0.1^{\circ} \mathrm{C} ; \mathrm{p}=0.02\right)$ and remained lower during the early postoperative period. Forearm minus fingertip skin-surface temperature gradient (an index of peripheral vasoconstriction) was greater in the routine care group in the early postoperative period. The mean norepinephrine concentration $(\mathrm{pg} / \mathrm{ml})$ was higher in the routine care group immediately postoperative $(480 \pm 70 \mathrm{pg} / \mathrm{ml}$ versus $330 \pm 30 \mathrm{pg} / \mathrm{ml} ; \mathrm{p}=0.02$ ) while mean epinephrine concentrations were not 
significantly different between groups. The mean cortisol levels were increased in both groups during the early postoperative timeframe, but the differences between groups were not significant. Systolic, mean, and diastolic arterial blood pressures were significantly higher in the routine care group. Researchers concluded that hypothermic patients had a greater degree of peripheral vasoconstriction, higher blood pressures, and higher norepinephrine levels during the early postoperative phase of care and that these findings suggest cardiovascular morbidity may be related to hypothermia in the perioperative period (Frank et al., 1995).

In another study, Wenisch and others (1996) tested the hypothesis that mild intraoperative hypothermia decreases both phagocytic capacity and the generation of reactive oxygen intermediates in polymorphonuclear leukocytes. Random assignment was conducted with 10 surgical patients assigned to intraoperative core temperatures ranging from 33 to $37^{\circ} \mathrm{C}$. The production of reactive oxygen intermediates and neutrophil phagocytosis were measured using flow cytometry. Phagocytic capacity was evaluated by uptake of fluorescein isothiocyanate labeled with Escherichia coli. Reactive oxygen production was estimated by the intracellular conversion of dihydrorhodamine 123 to rhodamine 123. Arterial blood samples were taken preoperatively, one hour after surgery started and 2 hours postoperatively. Results showed that mild intraoperative hypothermia reduced the generation of oxygen-free radicals by more than $50 \%$ and neutrophil phagocytosis by $72 \%$ from preoperative levels. Serum cortisol increased during surgery, and increased more during the postoperative phase. However, there was no relationship between neutrophil phagocytosis or reactive oxygen intermediate production and serum concentrations of acute phase reactants or plasma cortisol levels. Production of reactive 
oxidative intermediates was closely linked to body temperature and found to be linear, varying approximately fourfold over a $4^{\circ} \mathrm{C}$ range of core temperatures. Researchers concluded that since neutrophil oxidative function is one of the important defenses against bacteria that cause typical surgical site infections, impaired neutrophil oxidative killing may be a contributing factor hypothermia-induced reduction in resistance to infection (Wenisch et al., 1996).

\section{Therapeutic Effects Of Prewarming}

There is recent research documenting the therapeutic effects of preoperatively warming surgical patients. Prewarming raises mean body temperature by increasing the energy content in the peripheral thermal compartment of the body. This is important because it is difficult to treat the core hypothermia that occurs immediately after induction of general and regional anesthesia due to an internal core-to-peripheral redistribution of body heat. Discussion of four of these studies provided evidence that prewarming is beneficial.

Fossum and colleagues (2001) determined the effect of prewarming for 30 minutes with FAW (treatment group; $\mathrm{n}=50$ ) versus warmed cotton blankets (control group; $\mathrm{n}=50$ ) on core body temperature. The treatment group had significantly higher temperatures $\left(35.97^{\circ} \mathrm{C} \pm 0.52\right)$ on arrival to $\mathrm{PACU}$ than did the control group $\left(35.54^{\circ} \mathrm{C} \pm\right.$ $0.5 ; \mathrm{p}<.001)$. Of interest, the control group of patients also self-reported a higher incidence of shivering and thermal discomfort (Fossum, Hays, \& Henson, 2001).

In a prospective, randomized and blinded study by Vanni and others (2003), 30 patients undergoing abdominal surgery were placed in one of three groups according to the thermal management administered. In 10 patients, no special precautions were used to 
avoid hypothermia, whereas the other two groups received both preoperative and intraoperative active warming $(\mathrm{n}=10)$ or only intraoperative active warming $(\mathrm{n}=10)$. In the group that received only prewarming for 60 minutes, mean skin $\left(3.7^{\circ} \mathrm{C}\right)$, mean body $\left(1.9^{\circ} \mathrm{C}\right)$ and core temperature $\left(1.0^{\circ} \mathrm{C}\right)$ increased significantly $(\mathrm{p}<.05)$ before induction of anesthesia. Intraoperatively, the core temperature of prewarmed patients remained significantly higher $\left(36.2^{\circ} \mathrm{C} \pm 0.5^{\circ} \mathrm{C} ; \mathrm{p}<.05\right)$ than that of the other two groups. All patients warmed intraoperatively were normothermic at the end of surgery. In contrast, all unwarmed patients were hypothermic at the end of surgery and 5 of those patients suffered from postoperative shivering (Vanni, Braz, Modolo, Amorim, \& Rodrigues, 2003).

In a randomized, pretest/posttest experiment conducted to compare the effects of two preoperative warming approaches on perceptions of thermal comfort and anxiety, 118 preoperative patients were placed either in a forced-air warming (FAW) group or routine care (RC) group . The FAW group $(\mathrm{n}=60)$ had significantly higher thermal comfort levels in relation to their overall body temperature $(\mathrm{p}=.016)$, shivering $(\mathrm{p}=.010)$, and chest warmth $(\mathrm{p}=.003)$ in comparison to the $\mathrm{RC}$ group $(\mathrm{n}=58)$. Of interest, only the FAW group experienced a significant reduction in preoperative anxiety $(\mathrm{t}=2.77, \mathrm{p}=.007)$ compared to the $\mathrm{RC}$ group $(\mathrm{t}=.790, \mathrm{p}=.431)$. The researchers suggested that preoperative warming is a positive nursing intervention for both temperature management and anxiety reduction .

Melling, Ali, Scott and Leaper (2001) conducted a randomized controlled study investigating the effects of preoperative warming on the incidence of SSI after clean surgery. The sample included 416 patients undergoing elective hernia repair, varicose 
vein surgery or breast surgery that would result in a scar longer than $3 \mathrm{~cm}$ in length. Random assignment occurred with 139 assigned to standard treatment (non-warmed), 138 allocated to local warming (wound area warmed with radiant heat), and the last 139 patients assigned to systemic warming (forced air warming to whole body). Warming interventions lasted at least 30 minutes with staff nurses applying warming devices. Demographics and surgical variables were documented with core temperatures before treatment, after any warming, and after surgery. A single observer, unaware of treatment allocation, reviewed all patients at 2 and 6 weeks postoperative. He observed wounds and interviewed patients for wound history. Wounds were swabbed for microbiological analysis if purulent drainage was present at time of observation. Wounds were classified as infected if there was pus or a painful erythema that lasted for at least 5 days and was treated with antibiotics within the 6 weeks of surgery. The overall rate of SSI was $8 \%$ with a statistically higher rate of wound infection in the non-warmed group (14\%) compared to the combined warmed groups $(5 \% ; \mathrm{p}=.001)$. There was also statistical significance on the rate of wound infection when the two warmed groups were considered individually (systemic $\mathrm{p}=.026$; local $\mathrm{p}=.003$ ). The researchers concluded that prewarming patients, if done according to manufacturer's instructions, appears to have no adverse side effects. There were no statistical differences in outcome between the two types of warming interventions, although local warming resulted in $2 \%$ fewer infections. Of interest, the suggestion was made by the researchers that the hour before surgery may be of equal importance as the 'decisive period' of during and immediately postoperative because of the prevention of vasoconstriction by preoperative warming intervention (Melling, Ali, Scott, \& Leaper, 2001). 


\section{Discussion}

The need to undertake more human outcome studies was apparent during the literature review. It was also apparent that there are numerous differences in design, definitions of hypothermia, site and method of temperature measurement, and numerous other confounding variables related to the relationship between perioperative hypothermia and surgical stress. The literature review measurements of cytokines, catecholamines, and acute phase proteins, did not provide a solid basis from which to compare biologic values, especially from a preoperative perspective. Perhaps conduct of repeat studies to see if evidence is reproducible is another approach that could be taken, as that was not evident in this review of literature Future studies need to show sufficient power through large enough sample sizes, and develop reproducible methodological approaches in order to detect clinically important outcomes. The preoperative phase of care was not a focus in studies reviewed, therefore it would be relevant to suggest that future studies examine changes that occur preoperatively and even target early preoperative warming as an intervention against the risk of perioperative hypothermia.

There were numerous studies that discussed complications that have been associated with unplanned perioperative hypothermia, such as shivering, increased cardiac morbidity, coagulopathy, enhanced drug effects, venous stasis, and delayed recovery from anesthesia. This long list of complications alone should warrant the perioperative warming of all surgical patients, however, the evidence was so spread out and slim amongst each of these complications, that patients are still suffering due to a lack of strong cause and effect quantification for each. 
Most of the evidence discussed above supported the hypotheses that preoperative warming reduces perioperative hypothermia as well as reduces surgical stress in patients. Perioperative warming is beneficial as it increases perfusion, oxygenation and cellular immune responses. Intraoperative warming during major surgeries is becoming more of a routine practice in many institutions; however, evidence reviewed in this paper suggests that all surgical procedures warrant perioperative warming beginning in the preoperative phase of care. It was also apparent that nursing intervention studies are needed to find new approaches that decrease surgical stress and thereby improve immunocompetence postoperatively.

\section{Conceptual Model}

The perioperative experience includes elements such as the fear of surgery, anesthetic medications, tissue injury, and unplanned hypothermia - to name a few. The immune system provides an excellent biologic model to generate and test hypotheses related to perioperative care issues. Considering that anesthesia and surgery have broad effects on the immune system and provoke proinflammatory as well as immunosuppressive responses, it is reasonable that prevention of possible intraoperative and postoperative complications could begin during the preoperative phase of the patient's surgical experience.

This study was a preliminary effort in determining the effect of prewarming on redistribution hypothermia and the initial acute phase response of surgical stress. The conceptual framework for this study, depicted in Figure 1, was based on the FAW intervention providing a moderating effect on unplanned hypothermia that would lead to decreased surgical stress. 
Figure 1: Conceptual Model

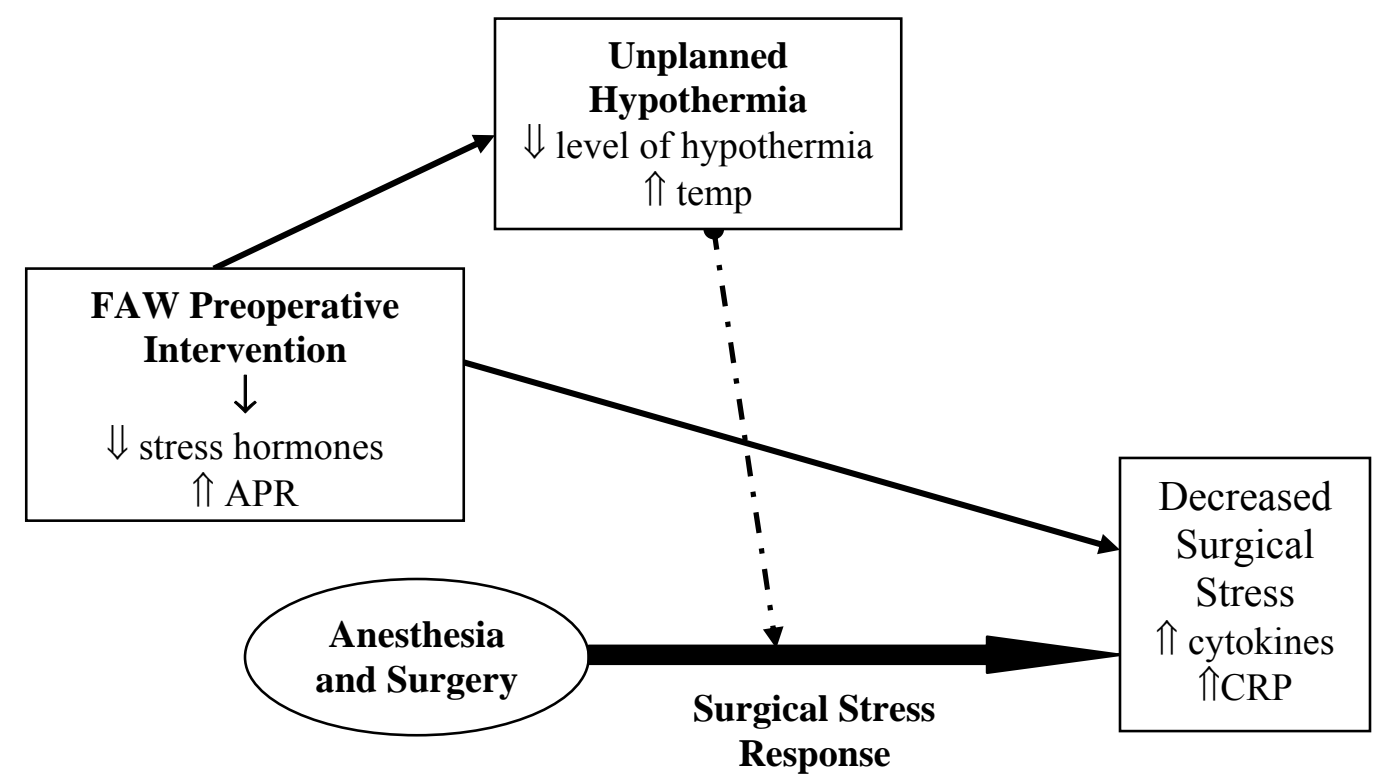

Note: The conceptual model illustrates the moderating effect of a preoperative FAW intervention on unplanned hypothermia and the acute phase response of surgical stress.

The perioperative immune system responds to multiple insults from the start of the surgical experience in order to preserve life, heal incised/excised tissues and fight infection. The FAW preoperative intervention was hypothesized as decreasing the catecholamine and cortisol levels of stress while increasing the healing processes of the initial acute phase response. Any continued stimulation of the stress response leads to possible reduced cardiac function, reduced immunocompetence, delayed wound healing, and a general loss of sufficient energy stores to promote healing (Edwards, 2003).

Preoperative FAW was theorized as one way to decrease surgical stress by allowing more normal temperature levels during surgery and thereby interacting with the stimulation of the stress response. 
The FAW intervention was also hypothesized to lessen the unplanned hypothermia that routinely occurs intraoperatively. The broken line coming from the unplanned hypothermia represents a decreased hypothermia from the FAW intervention and as an interaction with the surgical stress response. The patient that receives a FAW intervention would ultimately have decreased surgical stress demonstrated by higher proinflammatory cytokine and CRP production that would reflect a functional acute phase response intra- and postoperatively. This conceptual model reflects the intervention as an action intended to protect the body by ensuring an upregulation of the inflammatory response that acts to protect and heal without decreasing the competence of the immune system.

\section{Study Hypotheses}

This study was developed from research questions and directional hypotheses. In order to answer the research question, the following hypotheses were tested:

1) What is the effect of type of preoperative warming on the occurrence of intraoperative redistribution hypothermia?

Hypothesis 1: The FAW group will experience less unplanned perioperative hypothermia than the RC group.

2) What is the effect of type of preoperative warming on the acute phase response of surgical stress during the perioperative period Hypothesis 2: The FAW group will experience lower catecholamine and cortisol levels than the RC group.

3) Is there a difference in the level of surgical stress response between the FAW group of patients versus the routine care group of patients? 
Hypothesis 3: The FAW group will experience higher pro-inflammatory cytokine and CRP production intra and post-operatively than the RC group.

\section{Summary}

The literature review provided evidence of support for this preliminary study and the conceptual framework. This evidence was discussed from the perspective of temperature physiology, the etiology of unplanned hypothermia and the cellular responses to surgery and hypothermia, as well as the therapeutic effects of prewarming. The lack of nursing studies that focus on FAW as a preoperative intervention for decreasing surgical stress was recognized. The conceptual framework guided the development and organization of the study as well as led to the hypotheses used to answer the research questions posed at the start of the study process. This chapter provided discussion about the overall context for the phenomenon under study. 


\section{Chapter Three: Method}

This chapter describes the research plan and includes the research design, selection of setting, power analysis and sample size, and selection criteria for participants in this study. Institutional Review Board approval and the confidentiality and anonymity of participants' data are discussed. Data collection procedures and analysis methods are also found in this chapter.

\section{Research Design}

The method used was a randomized, equivalent group, experimental design to test the following hypotheses: 1) The FAW group will experience less unplanned perioperative hypothermia than the RC group. 2) The FAW group will experience lower catecholamine and cortisol levels than the RC group. 3) The FAW will experience higher pro-inflammatory cytokine and CRP production intra and post-operatively than the RC group.

The use of either an experimental or routine practice of preoperative warming intervention served as the independent variable. The effects of this manipulation on thermoregulation and acute phase stress response were examined. Confounding variables, such as ambient temperatures, blanket temperatures, surgery duration, and patient's physical status were measured on each patient. 


\section{Protection of Human Participants}

\section{Study Approval}

Approval for this study was obtained from the Institutional Review Board (IRB) of the University of South Florida (Appendix A: IRB Approval Letter) and verbal permission was received from the Research Committee at the surgical facility where the study was conducted.

Recruitment Process

The recruitment flyer was made available to all patients in the Preadmission and Testing Department minimized perception of coercion and the recruits were told that their participation was voluntary (Appendix B: Recruitment Flyer). The large print recruitment flyer briefly described the nursing study and asked for interested participants. If a patient stated an interest in participating in the study to their preadmissions nurse, then the investigator approached the patient to further describe the study, ascertain if the patient met inclusion criteria, and obtained informed consent.

\section{Informed Consent Process}

Written informed consent was obtained from each eligible participant prior to participation in the research process (Appendix C: Informed Consent Form). Understanding was assured by asking if the participant had questions and if the participant could verbalize accurately what he/she had agreed to do as a participant in the study.

\section{Benefits and Risks of Participation}

At this facility, different teams of health professionals see the patient at each phase of perioperative care. The main benefit of participation was the opportunity to have 
one familiar person (the nurse researcher) with them throughout their surgical experience.

Participants were withdrawn from the study when they requested to be withdrawn and at no time was the surgery delayed for the sake of the study. The forced-air warming blanket posed very low risk of thermal injury and is made of a non-woven medical fabric manufactured by Dupont. The blanket materials are latex free and biocompatible. There were no adverse events during the FAW interventions. The FAW blanket can make an occasional patient feel too warm, and when this occurred, the temperature of the air entering the blanket was adjusted. Core temperature monitoring also enabled the researcher to decrease the temperature of the blanket if overwarming of the participant occurred. There were two instances where the blanket airflow was too warm for the patient and the temperature was adjusted on one case and turned off earlier than the 30minute intervention in another case. These types of events were documented for statistical control.

Antecubital venipuncture was used to obtain eight tubes $(10 \mathrm{ml}$ and $4 \mathrm{~mL} \times 4$ collections) of venous blood and the participant was told that there could be mild discomfort and possibly a small amount of bruising. A separate intermittent IV access (INT) to use for all 4 blood draws was used to decrease multiple venipunctures for each participant. It was placed at the same time as the routine IV line in the preoperative setting. This decreased the amount of possible discomfort and bruising involved. Assessment of the site and removal of the access line was documented on each patient after the last blood sample was taken in PACU. 


\section{Sample Selection}

A power analysis was conducted during the planning of the study that showed a total of 116 participants with $50 \%$ in the FAW treatment group ( $\mathrm{n}=58)$, and $50 \%$ in the routine care group $(\mathrm{n}=58)$ would provide sufficient power. This power analysis was based on type I error rate of .05 , a type II error rate of .20 , and an effect size of $r=.30$ based on past research of a prewarming intervention study.

After the first set of assays were run and preliminary analysis was conducted on 9 participant samples in the laboratory, it was determined that high sensitivity assay plates were needed. Due to the increased cost of the different assay plates, it was cost prohibitive to continue with a large sample size. The decision to conduct a preliminary study with approximately 30 participants was made at that time. Figure 2 depicts the flow of participant status during the conduct of the study.

Figure 2 Flow Diagram of Participant Status During the Study

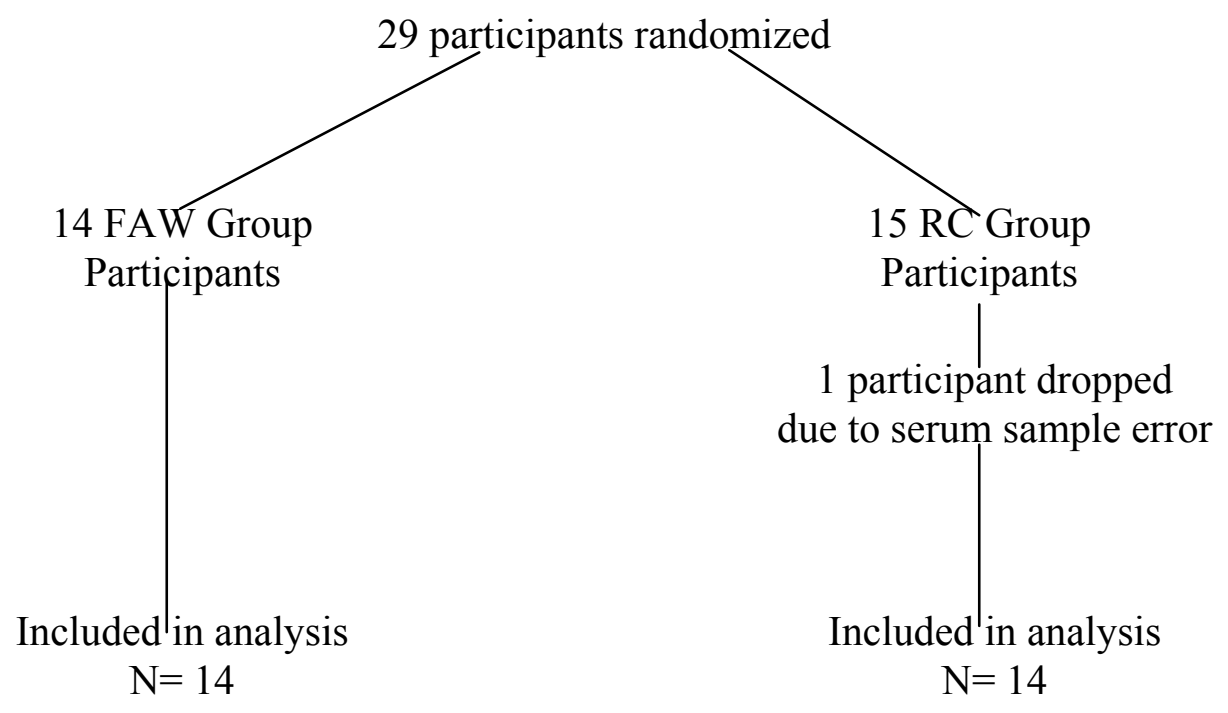




\section{Study Setting}

This study was conducted over a 5 and one half month period at a public, 98-bed acute care facility with 6 OR's. All patients coming into the preadmission and testing area were given a recruitment flyer. If a patient stated an interest in participating in the study, the investigator described the study, ascertained if the patient met inclusion criteria, and obtained informed consent.

Prior to starting the study, an inservice was given to the surgical services staff so they would be aware of the research and its purpose as well as their role in continuing to practice in their usual manner when involved in a study patient case. The surgeons and anesthesia providers were all given a letter describing the study (Appendix D: Surgeon Letter; Appendix E: Anesthesia Provider Letter). Verbal permission was obtained in order to use the hospital laboratory's centrifuge so blood samples could be handled appropriately for assay.

\section{Inclusion and Exclusion Criteria}

Inclusion criteria included any patient 18 to 65 years of age, competent and undergoing general anesthesia with a scheduled clean or clean-contaminated surgical procedure. Exclusion criteria included minimally invasive surgery involving scopes (e.g., arthroscopy, laparoscopy) and any patient exhibiting the following: paralysis or a known paresthesia, hyper/hypothyroidism, immunosuppression drug or radiation therapy within the past 6 months, febrile status (temperature $>38 \mathrm{C}^{\circ}$ ), and inability to tolerate tympanic temperatures. Non-English speaking patients were excluded due to the lack of interpreters. 


\section{Data Collection}

On the day of surgery and after arrival in the preoperative holding area, patients were assigned to one of two groups using a set of computer-generated random numbers. Demographic and health status data were gathered to control for confounding variables. The data collection forms are in Appendix F. A peripheral venous access device (IV line designated for blood samples) was inserted by the investigator, using hospital procedure, in order to decrease the amount of peripheral punctures for serum collection. The access site was placed in the opposite arm from the surgical IV access.

A minimum of four timed measurements throughout the perioperative period was recorded. The first set of measurements was recorded just before the warming intervention started and included vital signs with the core temperature taken by an infrared tympanic thermometer (Braun Thermoscan IRT 4520, Exac Temp). The following technique was used each time a temperature was taken was insertion of the probe into the ear, on the same side as the study IV access site, with the tip pointed toward the nose with the auricle pulled up and back. The ambient temperature was determined and recorded at each measurement with the use of an indoor/outdoor digital thermometer (Radio Shack Thermometer, Model 63-1009) that had a temperature range of $+32^{\circ}$ to $+122^{\circ} \mathrm{F}$. Two tubes $(10 \mathrm{~mL}$ serum separator and $4 \mathrm{~mL}$ plasma separator $)$ of venous blood were collected for measurement of CRP, pro-inflammatory cytokines, catecholamines, and cortisol levels. A second set of the same measurements was obtained at least 30 minutes after the start of intervention, and recorded before entering the OR suite and induction of anesthesia. The third blood sample and data measurements were taken 1 hour after induction of anesthesia. Upon arrival to the Post Anesthesia Care Unit 
(PACU), the investigator obtained the last set of measurements within a 30-minute time frame of admission to the PACU.

\section{Intervention Protocol}

Both groups put on a hospital gown and footies with a non-warmed cotton blanket placed over the legs for privacy prior to initial measurements being taken. After the initial set of measurements, those in the FAW group were covered with a forced-air warming blanket (Bair Hugger, model 111, Arizant Healthcare, Inc., Eden Prairie, MN) that connects to a warming unit (Bair Hugger ${ }^{\circledR}$, model 505) set on medium $\left(38^{\circ} \pm 3^{\circ} \mathrm{C}\right.$ or $\left.100.4 \pm 5.4^{\circ} \mathrm{F}\right)$ at that time. The warming equipment and blankets were donated and incurred no cost to the patient or facility. Once started, use of the warming blanket was continued for at least a 30 minute warming intervention.

After initial measurements were taken on the participants in the RC group, they were covered with one warmed cotton bath blanket. The blanket temperature was taken, at the time of placing it on the patient, with a handheld digital infrared thermal scanner

(OS951, Omega, Stamford, CT) that has an operating temperature range of 0 to $50 \mathrm{C}$ (32 to $122 \mathrm{~F}$ ) with only $1 \%$ reading emissivity error. The warmed cotton bath blanket was replaced twice at 15-minute intervals during the 30 minutes of intervention. Each replacement blanket had a documented thermal reading. The post intervention measurements were taken at least 30 minutes after the start of the control intervention.

\section{Serum Collection Protocol}

Two tubes of venous blood $(14 \mathrm{~m} / \mathrm{L})$ were collected for measurement of CRP, pro-inflammatory cytokines, catecholamines, and cortisol levels at each of the four collection times. All blood samples were placed on ice and transported to the lab within 
2 hours and processed for immediate freezing. The serum and plasma separator tubes were spun at $3500 \mathrm{rpm}$ for 10 minutes at $4^{\circ} \mathrm{C}$ in one of two hospital laboratory centrifuges (Beckman Spinchron $15 \mathrm{~N}-414880$ ), and the end products aliquoted into Eppendorf tubes and frozen at $-20^{\circ} \mathrm{C}$. Periodically, samples were shipped on dry ice to the USF biological laboratory for the investigator to analyze where they were stored at $80^{\circ} \mathrm{C}$ until analysis.

Assay Protocol

Analyses of biological variables were done in duplicate with appropriate controls and standards within 3 months of collection. For the cytokines (IL-1 $\beta$, IL-6, TNF- $\alpha$, \& IFN- $\gamma$ ), the samples were analyzed using a high sensitivity human cytokine LINCOplex 4-plex immunoassay kit utilizing Luminex ${ }^{\mathrm{TM}}$ technology. Luminex ${ }^{\mathrm{TM}}$ technology measures multiple analytes simultaneously in a single reaction vessel. Less than $50 \mu \mathrm{L}$ of sample is needed to test for these analytes at a single testing time. The assays are highly reliable and precise and were purchased as kits from Linco Research where this investigator's dissertation chairperson, Dr. Groer, was trained in the use of the Luminex analyzer. This investigator received training in the operation of the Luminex and was supervised by Dr. Groer.

The catecholamines, epinephrine and norepinephrine, were measured in plasma by research ELISA's (Rocky Mountain Diagnostics, Colorado Springs, CO) using a microtiter plate format with acylated catecholamines from the sample and the solid phase of the microtiter plate competing for a fixed number of antiserum binding sites. The antibody bound to the solid phase catecholamine was detected by an anti-rabbit IgGperoxidase conjugate using TMB as a substrate. The reaction was monitored at 450 
nanometers with the amount of antibody bound to the solid phase catecholamine being inversely proportional to the catecholamine concentration of the plasma sample.

Cortisol levels were measured by ELISA (DRG, Germany) with lower limits of detection at $2.5 \mathrm{ng} / \mathrm{mL}$ and high inter- and intra-assay specificity. The assay kit was a solid phase enzyme-linked immunosorbent assay based on the principle of competitive binding. The microtiter wells were coated with monoclonal antibody directed towards an antigenic site on the cortisol molecule. Endogenous cortisol of a patient sample competes with a cortisol-horseradish peroxidase conjugate for binding to the coated antibody. After incubation, the unbound conjugate was washed off and the amount of bound peroxidase conjugate was then inversely proportional to the concentration of cortisol in the serum sample. Absorbance was measured spectrophotometrically at 450 nanometers.

The C-reactive protein (DRG, Germany) in serum samples were measured using a high sensitivity assay system utilizing a unique monoclonal antibody directed against a distinct antigenic determinant on the CRP molecule. A mouse monoclonal anti-CRP antibody was used for solid phase immobilization. A goat anti-CRP antibody wass in the antibody-enzyme conjugate solution. The test sample was allowed to react simultaneously with the two antibodies, resulting in the CRP molecules being sandwiched between the sold phase and enzyme-linked antibodies. A tetramethylbenzidine (TMB) reagent was added and incubated for 20 minutes, resulting in the development of blue color. After development was stopped, the concentration of CRP was directly proportional to the color intensity of the test sample. Absorbance was then measured spectrophotometrically at 450 nanometers. 
Data from the ELISAs were converted from optical densities to concentrations and analyzed by Graph Pad. The Luminex instrument converted and analyzed the fluorescence intensity of the cytokine concentrations in the samples and provided one mean value of the duplicate samples. For each assay, a standard curve was obtained through the measurement of a set of standard samples. The concentrations of the experimental samples were then determined by comparison with this standard curve. The raw assay data were then entered on the statistical spreadsheet with the other data for analysis.

\section{Data and Safety Monitoring}

All data was kept confidential with all data locked in a file cabinet in the researcher's office during the conduct of the study. Privacy was insured by the use of a code number and computer files were set up to require passcodes for access. Only the student PI and the Faculty Advisor have access to the data.

Consent forms were kept separate from data gathering forms during the conduct of the study. Consents and data were then stored in a locked file cabinet that will be kept for 5 years in the researcher's office at home. Computer files were also placed on a CD for storage. Shredding of the paper files and the CD will be completed at the end of 5 years.

Participant safety was monitored on an ongoing individual basis. Protective measures to ensure the participant's privacy are outlined on the consent form (Appendix C) and confidentiality was maintained. The participant was withdrawn from the study at any time they requested to be withdrawn. The surgical procedure was not delayed for the sake of the study. The use of inclusion and exclusion criteria for determining 
participation status of patients was another monitoring tool for maintaining participant safety and control.

Data collection tools were tested on 6 patients at the start of the study to confirm usability, clarity, and process orientation of the forms. Adjustments were minor to the collection tools. Documentation of research activities will be kept in a lab notebook for review purposes as well as data integrity.

Analyses of biological samples were done in duplicate with appropriate controls and standards within 3 months of collection. Thermometers, blanket warming units, and the FAW equipment were continually monitored for function and calibration. The monitoring information was documented in a lab notebook for ease in review and maintenance of data integrity.

\section{Data Analysis}

To test the hypotheses that the independent variable of a FAW intervention would impact the dependent variables of temperature and acute phase response across time and between groups, the statistical analysis plan was a $(2 \times 4)$ repeated measures ANOVA design. The 4 time points used for assessment were the following: before warming treatment (Time 1); after warming treatment (Time 2); one hour after induction of anesthesia (Time 3); and upon admission to the PACU (Time 4). Sample characteristics regarding demographic and health characteristics were gathered from the participants. Data was coded, entered and analyzed using Statistical Package for the Social Sciences version 15 (SPSS, Inc., Chicago, IL). All statistical tests were evaluated for significance at the .05 level. 


\section{Summary}

This chapter provided an overview of the research plan that included the design, participant protections, setting and sample, as well as selection criteria. A discussion about the data collection was provided through the use of specific protocols. A review of the data and safety monitoring processes as well as the data analysis method were also discussed. 


\section{Chapter Four: Results}

Analysis of the collected data led to a view of the effect of a FAW intervention compared to routine care on the initial acute phase response of surgical stress. This chapter discusses data preparation, the profile of study participants, statistical analyses procedures. The test results of each hypothesis are discussed based on statistical test

findings. The use of tables and figures display the raw data at each time point of measurement.

\section{Data Preparation}

All data entries were entered with frequent data verifications by visual comparison of the data printout against the original data collection sheets. All errors in entry were corrected at that time. Outliers were also inspected using frequency distributions and boxplots. There were no outliers found between groups.

Histograms of each timed temperature reading (Time 1 to Time 4) were used to examine distributions for normality. Time 1 and Time 2 were positively skewed whereas Time 3 and Time 4 were negatively skewed. Computation of t-tests for each value of skewness was done and there were no significant values found. The decision to use raw temperature data in analysis procedures was made. Table 1 displays the descriptive statistics calculated on temperature data. 
Table 1: Descriptive Statistics Calculations on Temperature Across Time

\begin{tabular}{lrrrr}
\hline Statistics $(\mathrm{N}=28)$ & T1 Temp & T2 Temp & T3 Temp & T4 Temp \\
\hline Mean & 98.3 & 98.3 & 97.4 & 97.5 \\
Standard Deviation & .6150 & .6566 & 1.0758 & 1.0167 \\
Skewness & .464 & .635 & -.552 & -.591 \\
Std. Error of Skewness & .441 & .441 & .441 & .441 \\
t-test of skewness & 1.052 & 1.439 & -1.251 & -1.340 \\
\hline
\end{tabular}

Missing data were also assessed among the biologic variables. One participant's blood samples were not labeled appropriately, so a listwise deletion of this participant's data was completed. Other missing biologic data points were valid missing values because of no serum/plasma available at that specific time frame due to technical difficulties or the inability to draw a blood sample at a particular time point.

Raw data from the biological assays were entered and a non-normal distribution was determined. Because these variables show their effects on a logarithmic scale, a log 10 transformation was conducted to achieve a normal distribution before repeated measures ANOVA were computed. However, for ease in discussing and comparing data, the raw data values were used to plot and explain mean values in the tables and figures displayed in this report.

\section{Profile of Study Sample}

There were no significant differences between the RC and FAW groups. Participants in this study $(\mathrm{N}=28)$ were basically healthy and not considered at risk for surgery by the assignment of a physical level 1 or 2 (P1 or P2), as designated by the 
American Society of Anesthesiologists (ASA) and used by the anesthesiologists routinely during this study. The participants were scheduled for either a clean or a cleancontaminated invasive surgical procedure. The types of surgery included gynecologic and genitourinary, hernia repair with mesh, nasopharyngeal, open reduction fracture repair, and laminectomy procedures. Women comprised $75 \%(n=21)$ of the entire sample. There were both African American $(\mathrm{n}=10)$ and Caucasian $(\mathrm{n}=19)$ participants in the sample, but no other racial groups responded to recruitment. Ten participants were hypertensive and 2 diabetic with even distribution between groups. A display of the baseline characteristics of the study participants is found in Table 2 .

Table 2: Participant Characteristics $(\mathrm{N}=28)$

\begin{tabular}{|c|c|c|c|c|}
\hline Characteristic & Min & Max & Mean & SD \\
\hline Age & 22 & 64 & 44 & 11 \\
\hline Height & 61 & 76 & 66.5 & 3.4 \\
\hline Weight & 125 & 340 & 194 & 50 \\
\hline BMI & 21 & 58 & 31 & 8 \\
\hline Anesthesia duration & 33 & 207 & 113 & 39 \\
\hline Surgery duration & 21 & 189 & 88 & 38 \\
\hline PACU duration & 45 & 100 & 66 & 16 \\
\hline
\end{tabular}

All participants routinely received preoperative anxiolysis with the medication midazolam usually within 15 minutes prior to being transported to the OR. All underwent balanced general anesthesia that routinely included fentanyl, propofol, and nitrous oxide 
and were intubated for the procedure. It was also routine practice to provide warmed IV fluids in all perioperative settings. Intraoperative FAW was provided for 17 out of the 28 participants ( $\mathrm{n}=8$ in RC group; $\mathrm{n}=9$ in FAW group) by Anesthesia Providers. The Anesthesia Providers were neither deterred nor encouraged to provide intraoperative FAW. There were a total of $9(32 \%)$ hypothermic participants at Time 4 with postoperative shivering documented on 3 patients. Two of the participants with shivering were in the RC group with the other participant in the FAW group.

Of particular interest, the overall body mass index (BMI) of the participants was $31 \pm 8$ with approximately $71 \%$ of the sample population in the overweight to obese range. There were only 8 participants noted as within normal range on the body mass index.

\section{Statistical Analyses}

A 2 (warming type) x 4 (Time 4) repeated measure ANOVA was conducted using a probability value of $\mathrm{p}=<.05$ to test the following hypotheses:

1. The FAW group will experience less unplanned perioperative hypothermia than the $\mathrm{RC}$ group.

2. The FAW group will experience lower catecholamine and cortisol levels than the $\mathrm{RC}$ group.

3. The FAW group will experience higher pro-inflammatory cytokine and CRP production intra and post-operatively than the RC group.

Pearson product moment correlation coefficients were also calculated to determine relationships between temperatures, catecholamines, cortisol, CRP and 
cytokines, as well as other confounding variables, such as BMI and ambient temperatures.

\section{Test Assumptions}

The assumption of normal distribution of the dependent variables was reviewed and using a log 10 transformation of the biological data, that assumption was met. With repeated measures, there is correlation between the measures because they are from the same people, so the assumption of sphericity must be met. Specifically, the equality of variances of the differences between treatment levels in this experimental study were tested by Mauchly's Test, which tests the hypothesis that the variances of the differences between conditions are equal (Field, 2005). If the test statistic was significant $(p=<.05)$ then there were significant differences between the variances of differences and the condition of sphericity was not met. When the assumption of sphericity was violated, then the Greenhouse-Geisser correction was used.

Levene's Test was used to test for the assumption of equality of error variances between groups. When this test is non-significant, it indicates the assumption was met. When pairwise differences among treatment levels for a main effect was assessed, the Bonferroni post-hoc procedure was performed with a $p=.008$ level of significance.

\section{Hypotheses, Statistical Procedures, and Results}

\section{Hypothesis 1}

Hypothesis 1 predicted that the group of participants that received FAW would experience less unplanned perioperative hypothermia than the group of participants that received routine care. The results of a repeated measures ANOVA test showed a significant effect over time $(\mathrm{F}(3,78)=19.374, \mathrm{p}=<.001)$, a non-significant effect 
within subjects $(\mathrm{F}(1,26)=1.439, \mathrm{p}=.241)$, and a non-significant interaction effect $(\mathrm{F}$ $(3,78)=.390, p=.656)$. Since there was a significant effect of temperature across time, pairwise comparisons were analyzed. The mean difference $(\Delta \mathrm{M})$ between temperatures from Time 1 and $3(\Delta \mathrm{M}=.964, \mathrm{SE}=.191, \mathrm{p}=<.001)$ and Time 2 and $3(\Delta \mathrm{M}=.939, \mathrm{SE}$ $=.190, \mathrm{p}=<.001)$ were found statistically significant. Further comparison analysis also revealed Time 1 and $4(\Delta \mathrm{M}=.864, \mathrm{SE}=.184, \mathrm{p}=<.001)$ as well as Time 2 and $4(\Delta \mathrm{M}=$ $.839, \mathrm{SE}=.188, \mathrm{p}=.001)$ to be statistically significant. Figure 3 as well as Table 3 shows the descriptive statistics for each group at the four time points.

Figure 3: Mean Temperature Values $\left(\mathrm{F}^{\circ}\right)$ Across Time

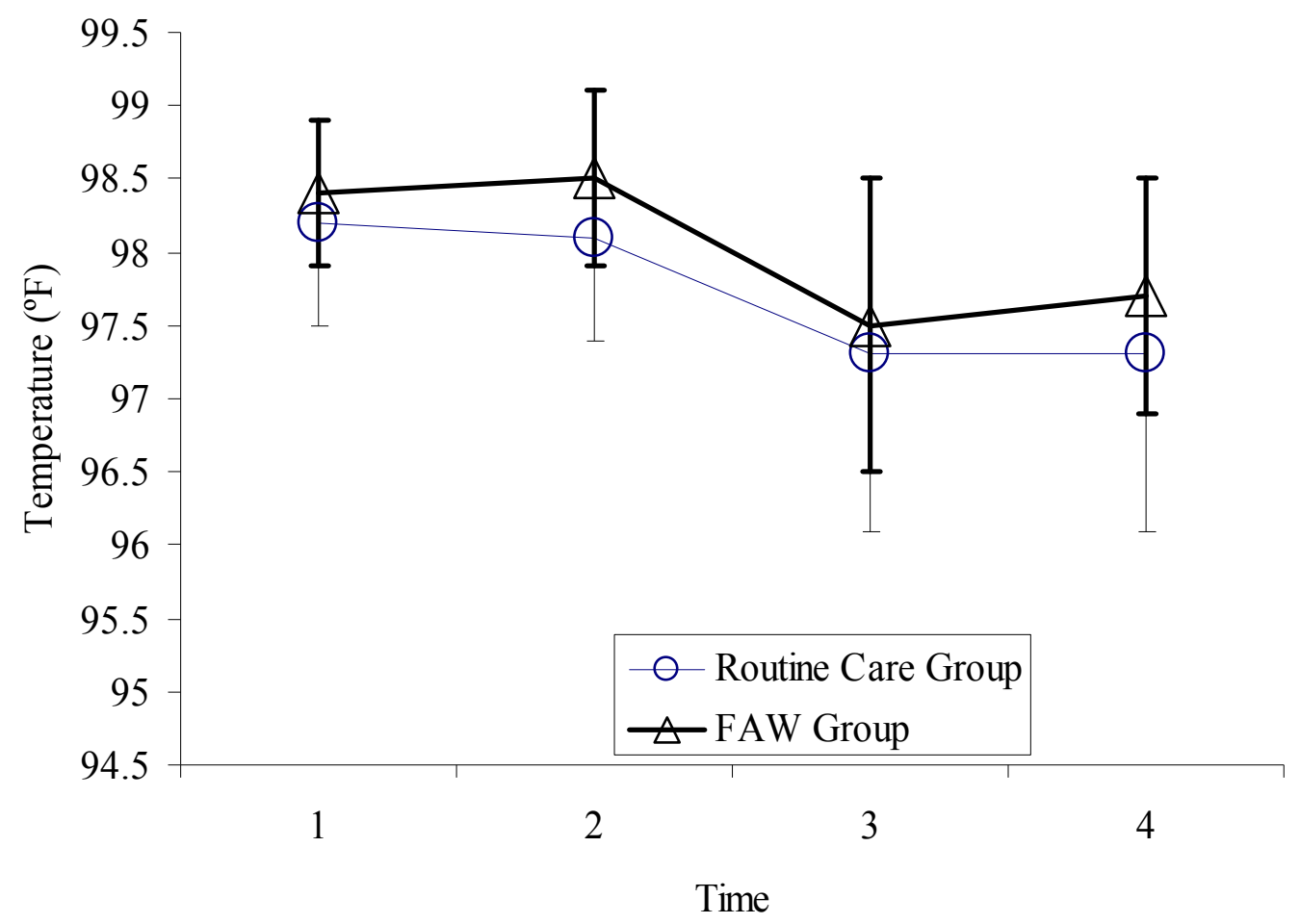

There were neither significant differences nor correlations between groups related to length of anesthesia, surgery duration, or ambient temperatures. Since the statistical 
analysis of interaction was not significant, this evidence showed a lack of statistical support for the first hypothesis.

Table 3: Descriptive Statistics of Temperatures $\left(\mathrm{F}^{\circ}\right)$ Across Time

\begin{tabular}{llcc}
\hline Time Reading of Temperature & \multicolumn{1}{c}{ Group Assignment } & $\begin{array}{c}\text { Mean } \\
\left(\mathrm{F}^{\circ}\right)\end{array}$ & $\begin{array}{c}\text { Std. } \\
\text { Deviation }\end{array}$ \\
\hline Time 1 temperature & RC group $(\mathrm{n}=14)$ & 98.2 & .7 \\
& FAW group $(\mathrm{n}=14)$ & 98.4 & .5 \\
& Total Mean & 98.3 & .6 \\
Time 2 temperature & RC group $(\mathrm{n}=14)$ & 98.1 & .7 \\
& FAW group $(\mathrm{n}=14)$ & 98.5 & .6 \\
Time 3 temperature & Total Mean & 98.3 & .7 \\
& RC group $(\mathrm{n}=14)$ & 97.3 & 1.2 \\
& FAW group $(\mathrm{n}=14)$ & 97.5 & 1.0 \\
& Total Mean & 97.4 & 1.1 \\
& RC group $(\mathrm{n}=14)$ & 97.3 & 1.2 \\
& FAW group $(\mathrm{n}=14)$ & 97.7 & .8 \\
& Total Mean & 97.5 & 1.0 \\
\hline
\end{tabular}

Hypothesis 2

Hypothesis 2 predicted that the FAW group would experience lower catecholamine and cortisol levels than the RC group. The results of repeated measures ANOVA testing on cortisol, epinephrine, and norepinephrine will be presented separately. 
Cortisol. There were no significant findings over time $(\mathrm{F}(3,75)=.272, \mathrm{p}=.771)$, within subjects $(\mathrm{F}(1,25)=.003, \mathrm{p}=.959)$, or between groups $(\mathrm{F}(3,75)=.073, \mathrm{p}=.935$. There were no relative correlations for the Cortisol measurements across time. Table 4 and Figure 4 display the raw mean values of Cortisol across time. These results did not support the $2^{\text {nd }}$ hypothesis.

Table 4: Raw Mean Values of Cortisol (ng/ml) Across Time

\begin{tabular}{|c|c|c|c|}
\hline Time & Group Assignment & Mean (ng/ml) & Std. Deviation \\
\hline \multirow[t]{2}{*}{ Cortisol Time 1} & RC group $(n=13)$ & 127.85 & 104.32 \\
\hline & FAW group $(n=14)$ & 132.16 & 117.06 \\
\hline \multirow[t]{2}{*}{ Cortisol Time 2} & $\mathrm{RC}$ group $(\mathrm{n}=13)$ & 151.64 & 153.08 \\
\hline & FAW group $(n=14)$ & 146.31 & 124.64 \\
\hline \multirow[t]{2}{*}{ Cortisol Time 3} & $\mathrm{RC}$ group $(\mathrm{n}=13)$ & 160.08 & 140.54 \\
\hline & FAW group $(n=14)$ & 132.81 & 93.47 \\
\hline \multirow[t]{2}{*}{ Cortisol Time 4} & $\mathrm{RC}$ group $(\mathrm{n}=13)$ & 135.56 & 91.59 \\
\hline & FAW group $(n=14)$ & 151.96 & 124.41 \\
\hline
\end{tabular}


Figure 4: Raw Mean Values of Cortisol (ng/ml) Across Time

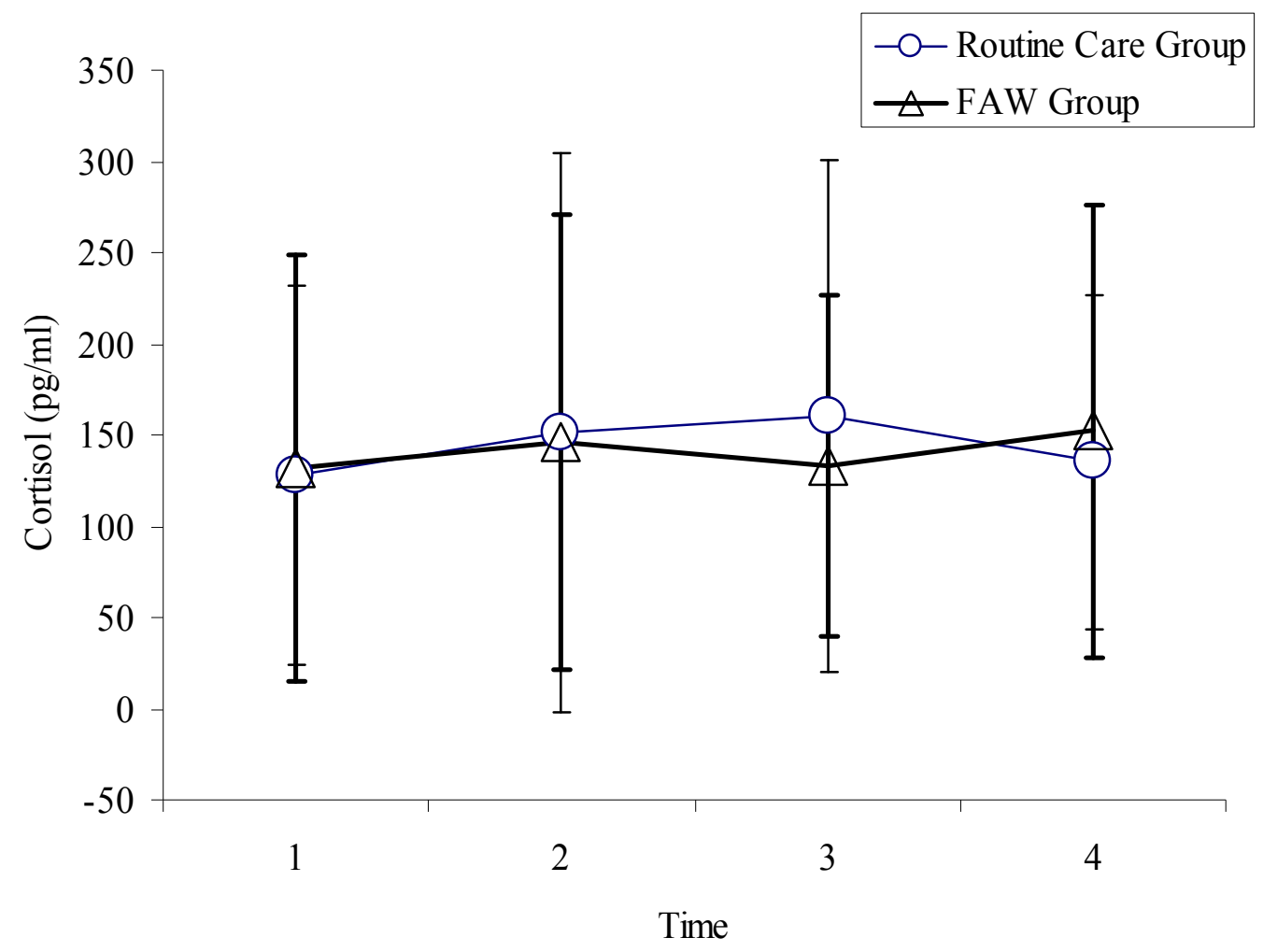

Epinephrine. There were incomplete sets of data $(\mathrm{n}=9)$ for analysis in the routine care group compared to the FAW group $(n=14)$ due to missing data at different time points. The results of a repeated measures ANOVA test for transformed epinephrine data showed a significant effect over time $(\mathrm{F}(3,63)=7.874, \mathrm{p}=.001)$, a non-significant within group effect $(\mathrm{F}(1,21)=.500, \mathrm{p}=.487)$ as well as no significant interaction effect $(F(3,63)=.068, p=.940)$. Since there was a significant effect of epinephrine across time, pairwise comparisons were analyzed. However, there were no statistically significant differences in the results. Table 5 illustrates the raw values of epinephrine activity within groups and between groups across time. 
Table 5: Raw Mean Values of Epinephrine (pg/ml) Across Time

\begin{tabular}{clcc}
\hline Time & Group Assignment & Mean $(\mathrm{pg} / \mathrm{ml})$ & Std. Deviation \\
\hline Epinephrine Time 1 & Routine Care group $(\mathrm{n}=9)$ & 41.01 & 31.47 \\
& FAW group $(\mathrm{n}=14)$ & 58.98 & 67.84 \\
Epinephrine Time 2 & Routine Care group $(\mathrm{n}=9)$ & 27.40 & 25.44 \\
& FAW group $(\mathrm{n}=14)$ & 37.04 & 26.48 \\
Epinephrine Time 3 & Routine Care group $(\mathrm{n}=9)$ & 68.52 & 55.53 \\
& FAW group (n=14) & 72.21 & 54.57 \\
& & & \\
Epinephrine Time 4 & Routine Care group (n=9) & 128.70 & 120.40 \\
& FAW group (n=14) & 121.57 & 85.03 \\
& & & \\
\hline
\end{tabular}

There were no significant correlations between epinephrine and the other variables of interest. Overall, the presentation of data showed the same type of catecholamine activity within and between groups. This data did not provide statistical support for hypothesis 2 . Figure 5 shows the raw values of epinephrine activity within subjects and between groups across time 
Figure 5: Raw Mean Values of Epinephrine (pg/ml) Across Time

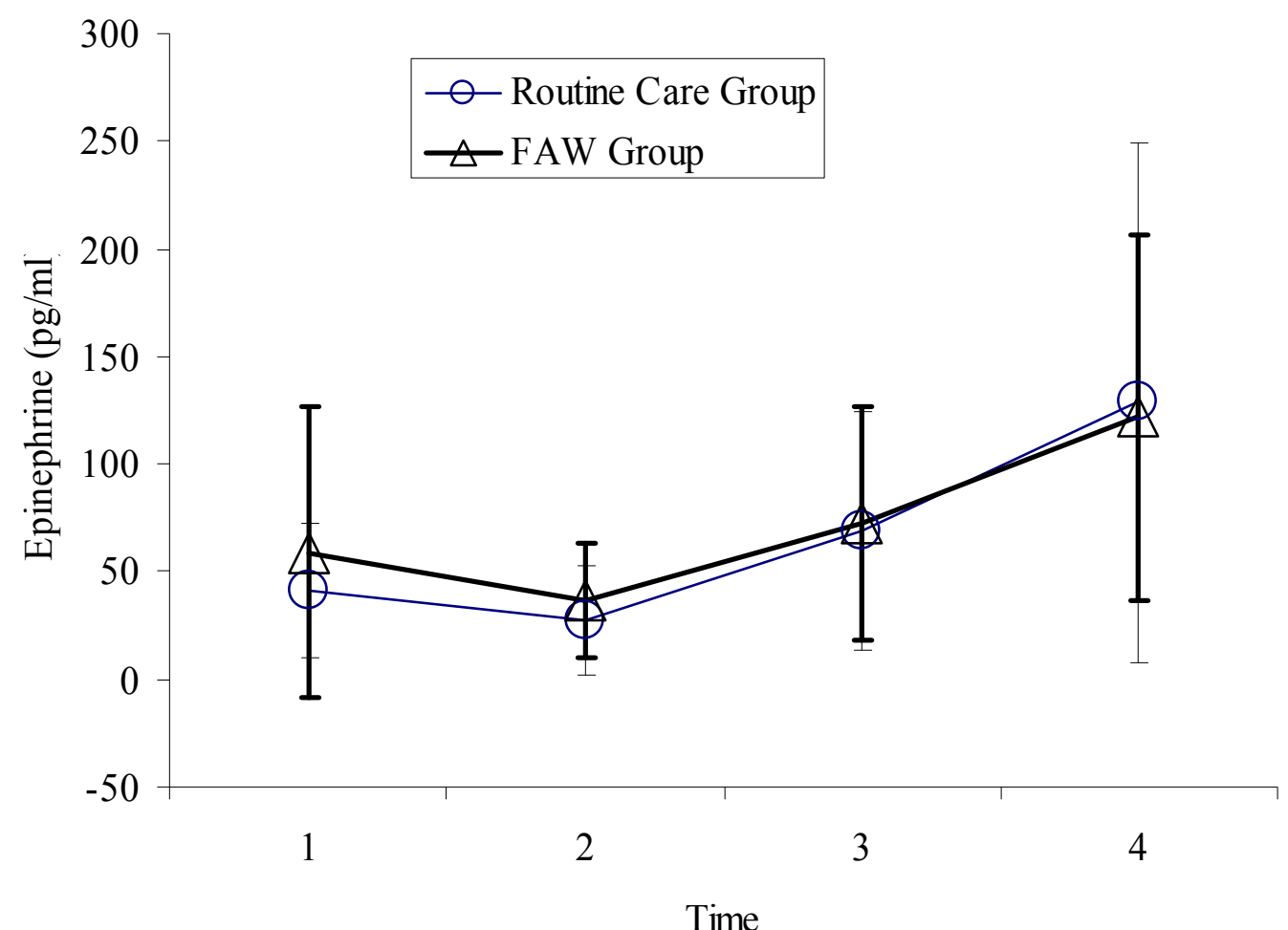

Norepinephrine. Again, there were not complete sets of data $(\mathrm{n}=10)$ for analysis in the routine care group when compared to the FAW group $(n=14)$. The results of a repeated measures ANOVA test for norepinephrine showed a significant effect over time $\mathrm{F}(3,66)=5.580, \mathrm{p}=.005)$, non-significance within groups $(\mathrm{F}(1,22)=.190, \mathrm{p}=.667)$ and no statistically significant interaction effect $(F(3,66)=1.300, p=.283)$. Pairwise comparisons were analyzed because there was a significant effect of epinephrine across time. The only pairwise comparison that was statistically significant was from Time 2 and $3(\Delta \mathrm{M}=-.245, \mathrm{SE}=.061, \mathrm{p}=.004)$.

Table 6 and Figure 6 illustrate the levels of norepinephrine activity within groups and between groups across time. This statistical analysis did not support the hypothesis. 
Table 6: Raw Mean Values of Norepinephrine (pg/ml) Activity Across Time

Time Reading of Mean

Std.

Norepinephrine

Group Assignment

$(\mathrm{pg} / \mathrm{ml})$

Deviation

Time 1

Routine Care group $(\mathrm{n}=10)$

216.91

171.96

FAW group $(\mathrm{n}=14)$

202.22

191.46

Time 2

Routine Care group $(\mathrm{n}=10)$

159.85

181.36

FAW group $(\mathrm{n}=14)$

169.34

163.53

Time 3

Routine Care group $(\mathrm{n}=10)$

253.61

300.77

FAW group $(\mathrm{n}=14)$

320.67

295.31

Time 4

Routine Care group $(\mathrm{n}=10)$

263.41

FAW group $(\mathrm{n}=14)$

509.81

432.57

Figure 6: Raw Mean Values of Norepinephrine (pg/ml) Across Time

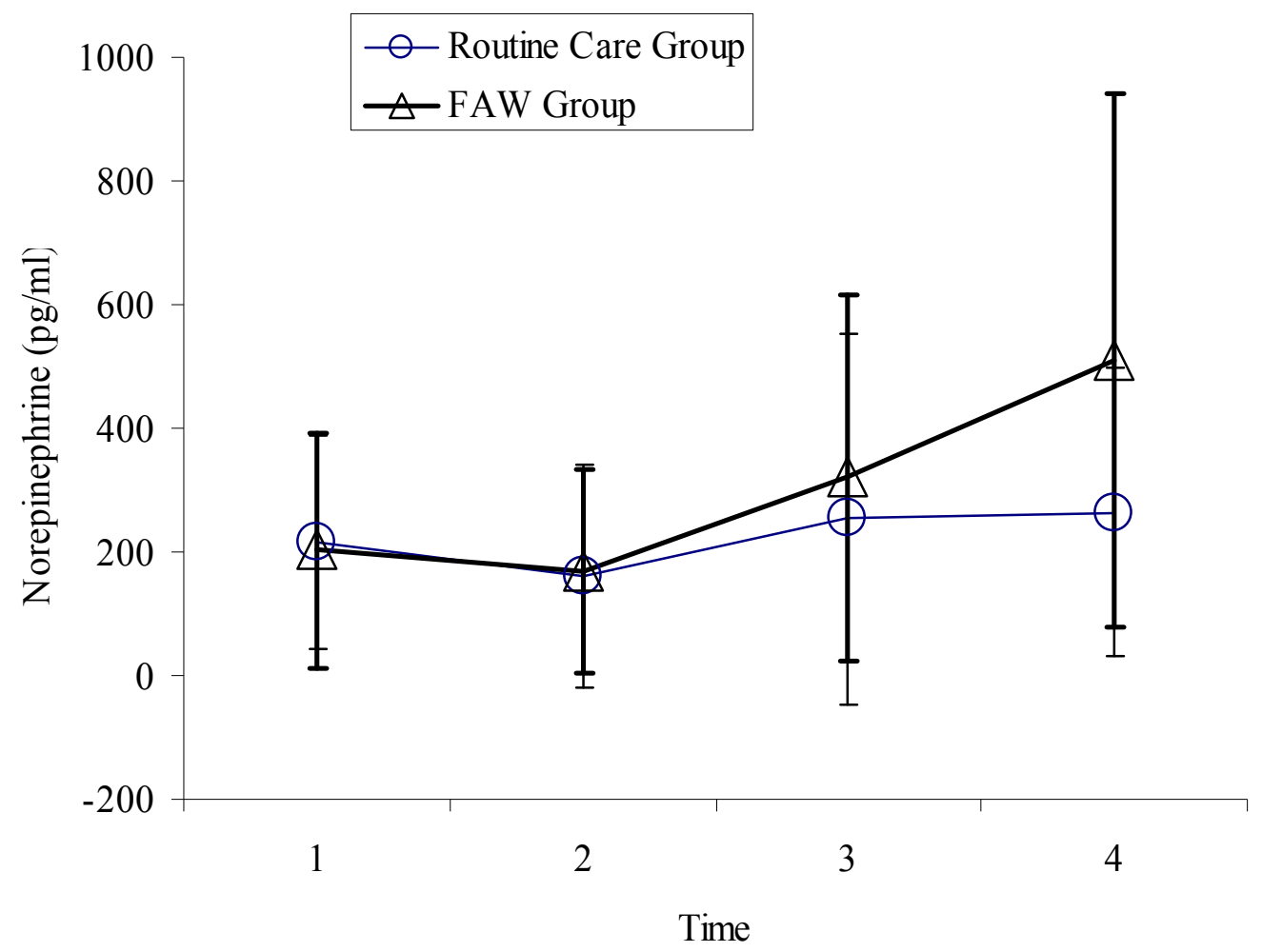




\section{Hypothesis 3}

Hypothesis 3 predicted that the FAW group would experience higher proinflammatory cytokine and CRP production intra and post-operatively than the RC group. The results of a repeated measures ANOVA tests on IL-1ß, IL-6, TNF- $\alpha$, IFN- $\gamma$, and CRP will be presented separately. The Time 1 upregulation of the cytokines were recognized as an item of interest and will be discussed in the next chapter.

$I L-1 \beta$. For the measure of IL-1ß, there were no statistically significant main effects $(F(3,78)=2.348, p=.124)$, between subjects effects $(F(1,26)=2.446, p=$ $.130)$, or interaction effects $(\mathrm{F}(3,78)=1.407, \mathrm{p}=.253)$. The predicted hypothesis was not supported by the measurement values of IL-1ß. Table 7 and Figure 7 show the raw mean values of IL-1ß between groups over time.

Table 7: Raw Mean Values of IL-1ß (pg/ml) Across Time

\begin{tabular}{|c|c|c|c|}
\hline Time & Group Assignment & $\begin{array}{c}\text { Mean } \\
(\mathrm{pg} / \mathrm{ml})\end{array}$ & $\begin{array}{c}\text { Std. } \\
\text { Deviation }\end{array}$ \\
\hline \multirow[t]{2}{*}{ IL-1 $\beta$ Time 1} & Routine Care group $(\mathrm{n}=14)$ & 5.78 & 3.43 \\
\hline & FAW group $(n=14)$ & 7.36 & 3.45 \\
\hline \multirow[t]{2}{*}{ IL-1 $\beta$ Time 2} & Routine Care group $(\mathrm{n}=14)$ & 5.30 & 3.61 \\
\hline & FAW group $(n=14)$ & 6.72 & 3.65 \\
\hline \multirow[t]{2}{*}{ IL-1 $\beta$ Time 3} & Routine Care group $(n=14)$ & 5.43 & 3.51 \\
\hline & FAW group $(n=14)$ & 6.79 & 3.85 \\
\hline \multirow[t]{2}{*}{ IL-1 $\beta$ Time 4} & Routine Care group $(\mathrm{n}=14)$ & 4.51 & 3.53 \\
\hline & FAW group $(n=14)$ & 7.20 & 4.22 \\
\hline
\end{tabular}


Figure 7: Raw Mean Values of IL-1ß (pg/ml) Across Time

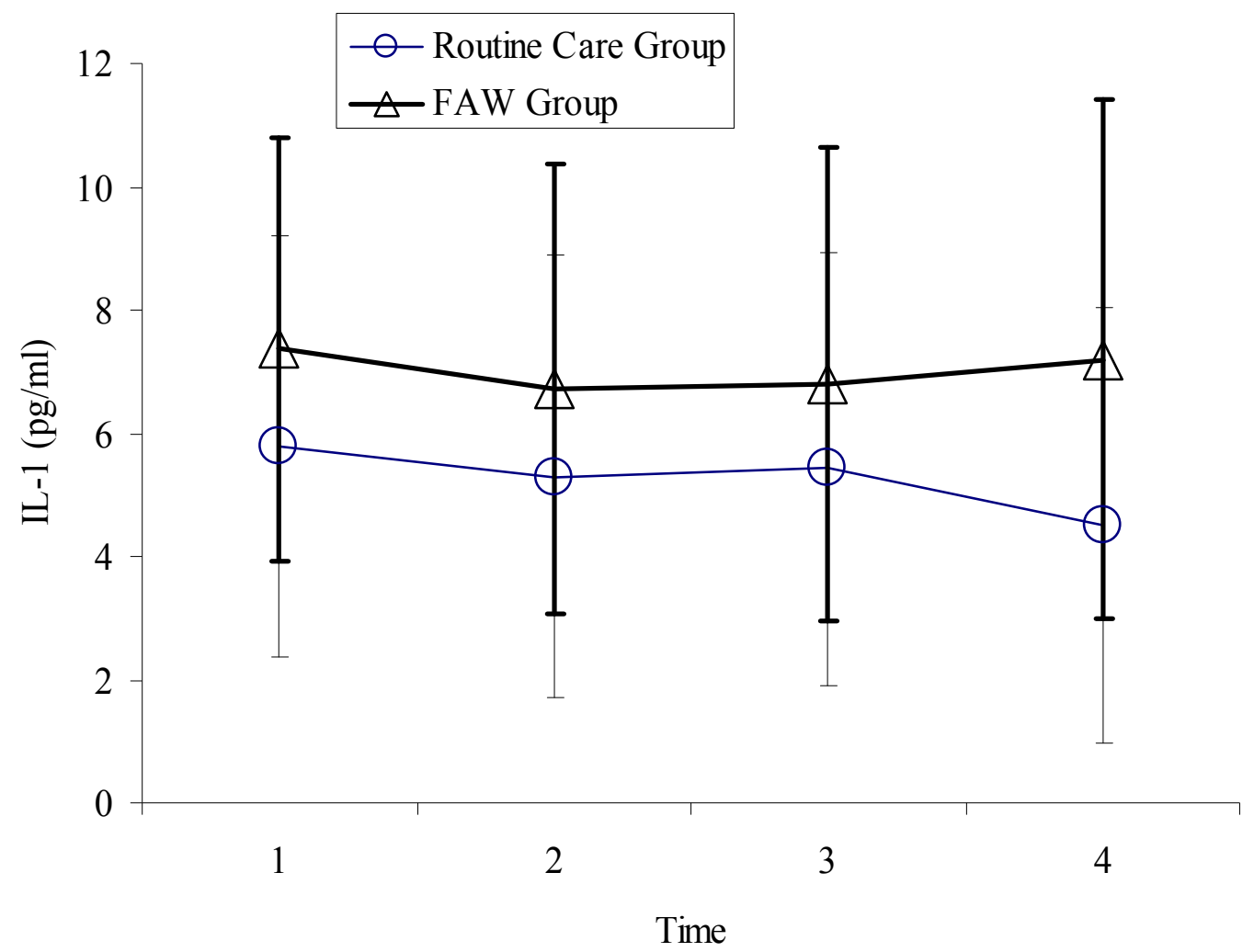

IL-6. The results of a repeated measures ANOVA test for IL-6 showed a significant effect over time $(\mathrm{F}(3,78)=8.403, \mathrm{p}=.004)$, no significant effect between subjects $(\mathrm{F}(1,26)=.951, \mathrm{p}=.339)$, and no significant interaction effect $(\mathrm{F}(3,78)=$ $2.593, p=.109)$. Since there was a significant effect of IL-6 across time, pairwise comparisons were analyzed. There were no statistically significant findings in that comparison data. Table 8 and Figure 8 show the raw mean values of IL- 6 between groups across time. Il-6 was correlated with TNF- $\alpha$ at times 1 through 3 . Table 9 displays the correlation coefficients for IL- 6 and TNF- $\alpha$ across time. 
Table 8: Raw Mean Values of IL-6 (pg/ml) Across Time

\begin{tabular}{llcc}
\hline Time & Group Assignment & $\begin{array}{c}\text { Mean } \\
(\mathrm{pg} / \mathrm{ml})\end{array}$ & $\begin{array}{c}\text { Std. } \\
\text { Deviation }\end{array}$ \\
\hline IL-6 Time 1 & Routine Care group $(\mathrm{n}=14)$ & 28.31 & 21.79 \\
& FAW group $(\mathrm{n}=14)$ & 25.06 & 18.24 \\
IL-6 Time 2 & Routine Care group $(\mathrm{n}=14)$ & 29.44 & 26.31 \\
& FAW group $(\mathrm{n}=14)$ & 22.97 & 18.12 \\
IL-6 Time 3 & Routine Care group $(\mathrm{n}=14)$ & 28.72 & 23.48 \\
& FAW group (n=14) & 23.85 & 17.52 \\
IL-6 Time 4 & Routine Care group $(\mathrm{n}=14)$ & 32.42 & 25.06 \\
& FAW group $(\mathrm{n}=14)$ & 31.35 & 15.73 \\
\hline
\end{tabular}

Table 9: Correlation Coefficients for IL-6 and TNF- $\alpha$ Across Time

\begin{tabular}{llrrrr}
\hline & N $=28$ & TNF T1 & TNF T2 & TNF T3 & TNF T4 \\
\hline IL-6 T1 & Pearson Correlation & $.381\left(^{*}\right)$ & $.377(*)$ & .277 & .256 \\
& Sig. (2-tailed) & .045 & .048 & .154 & .188 \\
IL-6 T2 & Pearson Correlation & .372 & $.401\left(^{*}\right)$ & .276 & .246 \\
& Sig. (2-tailed) & .051 & .034 & .155 & .208 \\
IL-6 T3 & Pearson Correlation & $.376(*)$ & $.386(*)$ & .299 & .249 \\
& Sig. (2-tailed) & .048 & .043 & .123 & .202 \\
IL-6 T4 & Pearson Correlation & .113 & .160 & .049 & .004 \\
& Sig. (2-tailed) & .566 & .417 & .804 & .985 \\
\hline
\end{tabular}

*Correlation is significant at the 0.05 level (2-tailed). 
Figure 8: Raw Mean Values of IL-6 (pg/ml) Across Time

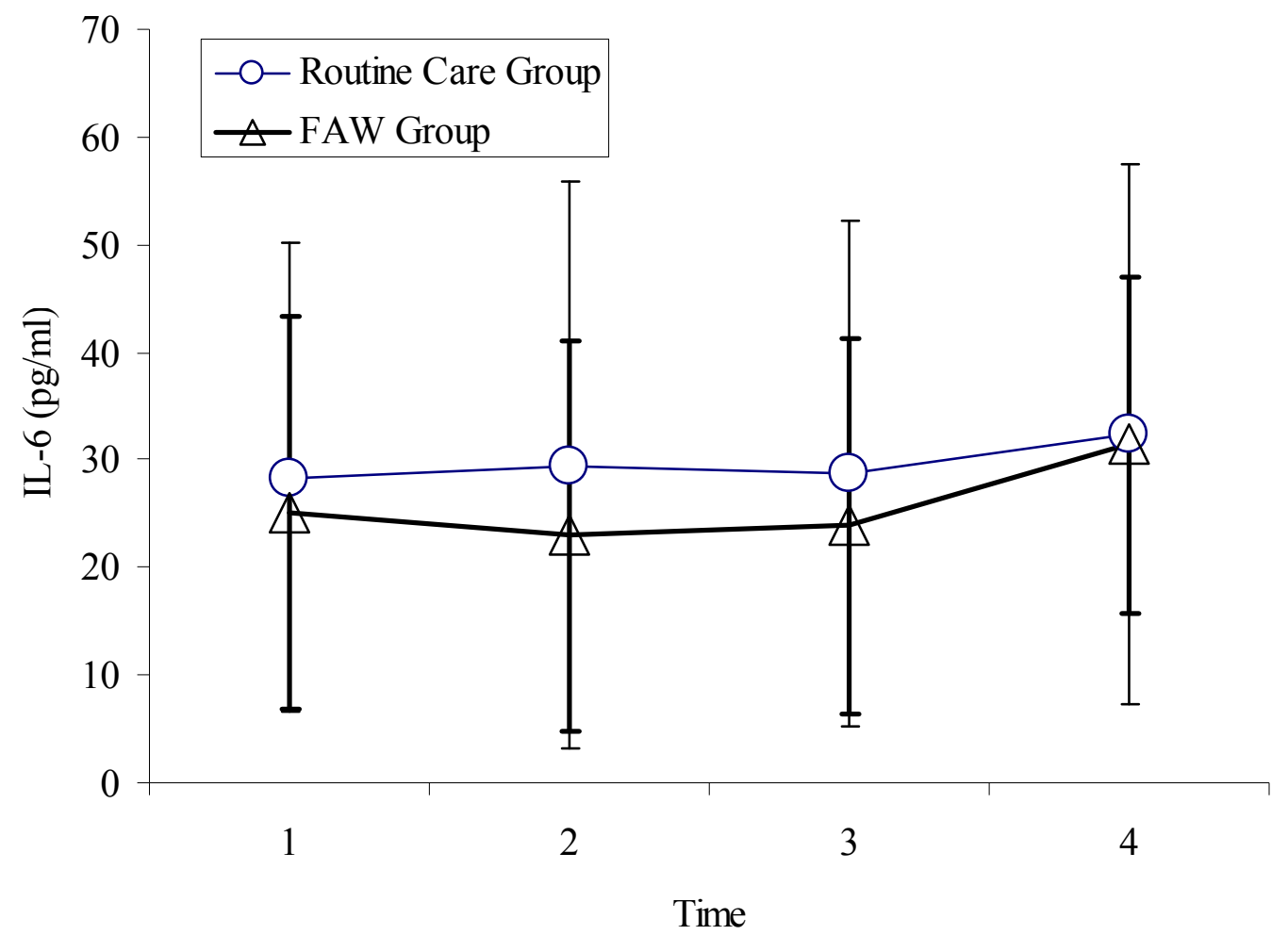

$T N F-\alpha$. The results of a repeated measures ANOVA test for TNF- $\alpha$ showed a significant effect over time $(\mathrm{F}(3,78)=4.309, \mathrm{p}=.007)$, a non-significant between subjects effect $(\mathrm{F}(1,26)=1.248, \mathrm{p}=.274)$, and no significant interaction effect $(\mathrm{F}(3$, $78)=1.847, p=.145)$. Since there was a significant effect of TNF- $\alpha$ across time, pairwise comparisons were analyzed. There were no statistically significant comparisons found in the comparisons analysis. Table 10 and Figure 9 display the raw mean values of TNF- $\alpha$ activity between groups across time. 
Table 10: Raw Mean Values of TNF- $\alpha$ Activity Across Time

\begin{tabular}{clcc}
\hline Time & Group Assignment & Mean & $\begin{array}{c}\text { Std. } \\
\text { Deviation }\end{array}$ \\
\hline TNF- $\alpha$ Time 1 & Routine Care group $(\mathrm{n}=14)$ & 6.45 & 2.03 \\
& FAW group $(\mathrm{n}=14)$ & 8.95 & 5.70 \\
TNF- $\alpha$ Time 2 & Routine Care group $(\mathrm{n}=14)$ & 6.38 & 2.52 \\
& FAW group $(\mathrm{n}=14)$ & 8.25 & 6.07 \\
TNF- $\alpha$ Time 3 & Routine Care group $(\mathrm{n}=14)$ & 6.74 & 2.82 \\
& FAW group ( $\mathrm{n}=14)$ & 8.50 & 5.73 \\
TNF- $\alpha$ Time 4 & Routine Care group $(\mathrm{n}=14)$ & 5.82 & 2.36 \\
& FAW group ( $=14)$ & 8.25 & 5.29 \\
\hline
\end{tabular}

Figure 9: Raw Mean Values of TNF- $\alpha$ Activity Across Time

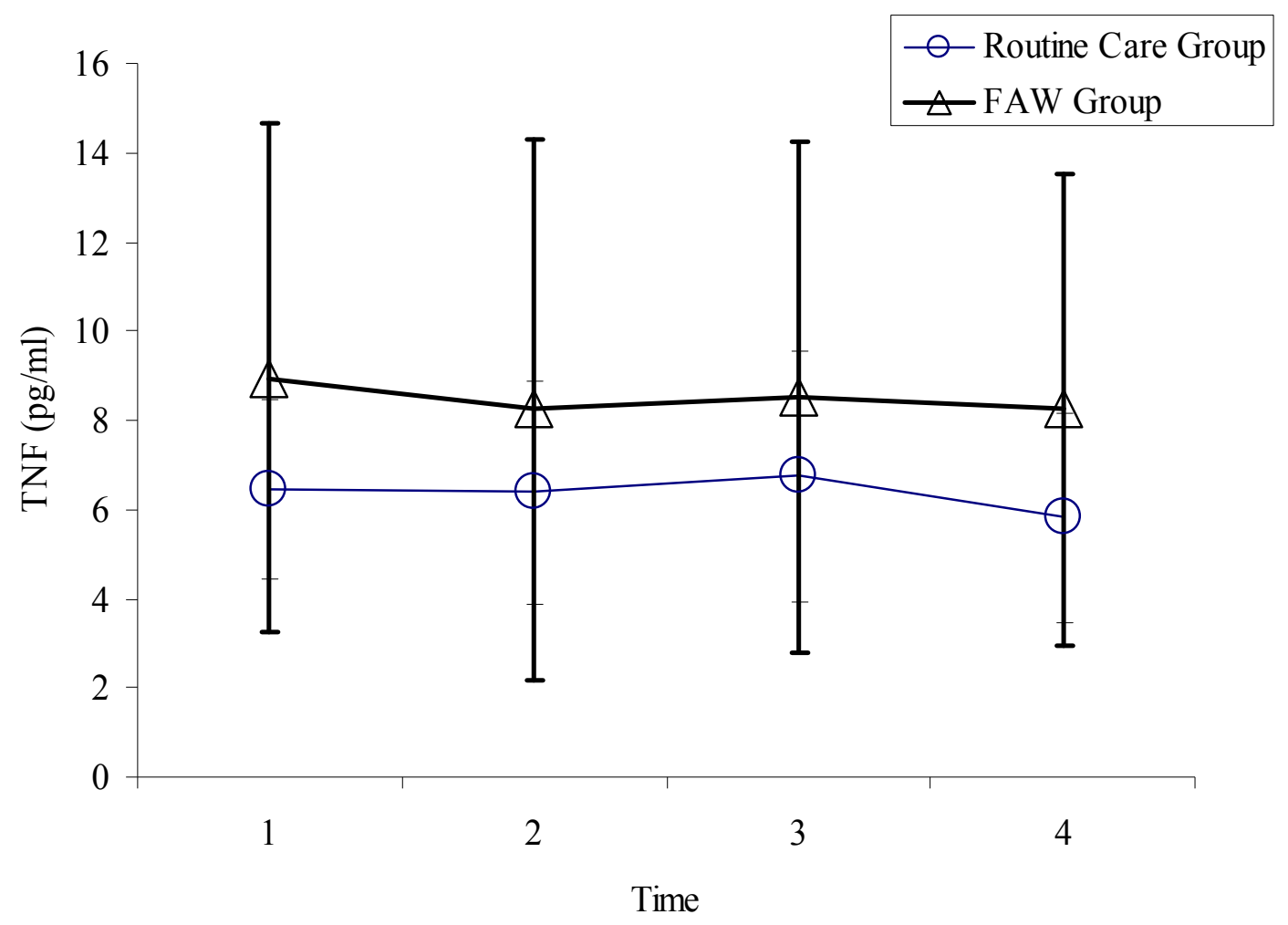


$I F N-\gamma$. For the measure of IFN- $\gamma$ there were no statistically significant main effects $(F(3,78)=2.591, p=.077)$, no significant effects between subjects $(F(1,26)=$ $.064, \mathrm{p}=.802)$, or interaction effects $(\mathrm{F}(3,78)=1.621, \mathrm{p}=.204)$. Table 10 and Figure 10 show the raw means of IFN- $\gamma$ activity between groups across time. No evidence was found to support the hypothesis.

Table 11: Raw Mean Values of IFN- $\gamma(\mathrm{pg} / \mathrm{ml})$ Across Time

\begin{tabular}{llcc}
\hline Time & Group Assignment & Mean & $\begin{array}{c}\text { Std. } \\
\text { Deviation }\end{array}$ \\
\hline IFN- $\gamma$ Time 1 & Routine Care group $(\mathrm{n}=14)$ & 44.24 & 39.90 \\
& FAW group $(\mathrm{n}=14)$ & 44.45 & 50.26 \\
IFN- $\gamma$ Time 2 & Routine Care group $(\mathrm{n}=14)$ & 39.82 & 40.27 \\
& FAW group $(\mathrm{n}=14)$ & 42.71 & 53.58 \\
IFN- $\gamma$ Time 3 & Routine Care group $(\mathrm{n}=14)$ & 41.38 & 38.69 \\
& FAW group $(\mathrm{n}=14)$ & 47.31 & 58.25 \\
IFN- $\gamma$ Time 4 & Routine Care group $(\mathrm{n}=14)$ & 33.10 & 31.91 \\
& FAW group $(\mathrm{n}=14)$ & 45.85 & 59.57 \\
\hline
\end{tabular}


Figure 10: Raw Mean Values of IFN- $\gamma(\mathrm{pg} / \mathrm{ml})$ Across Time

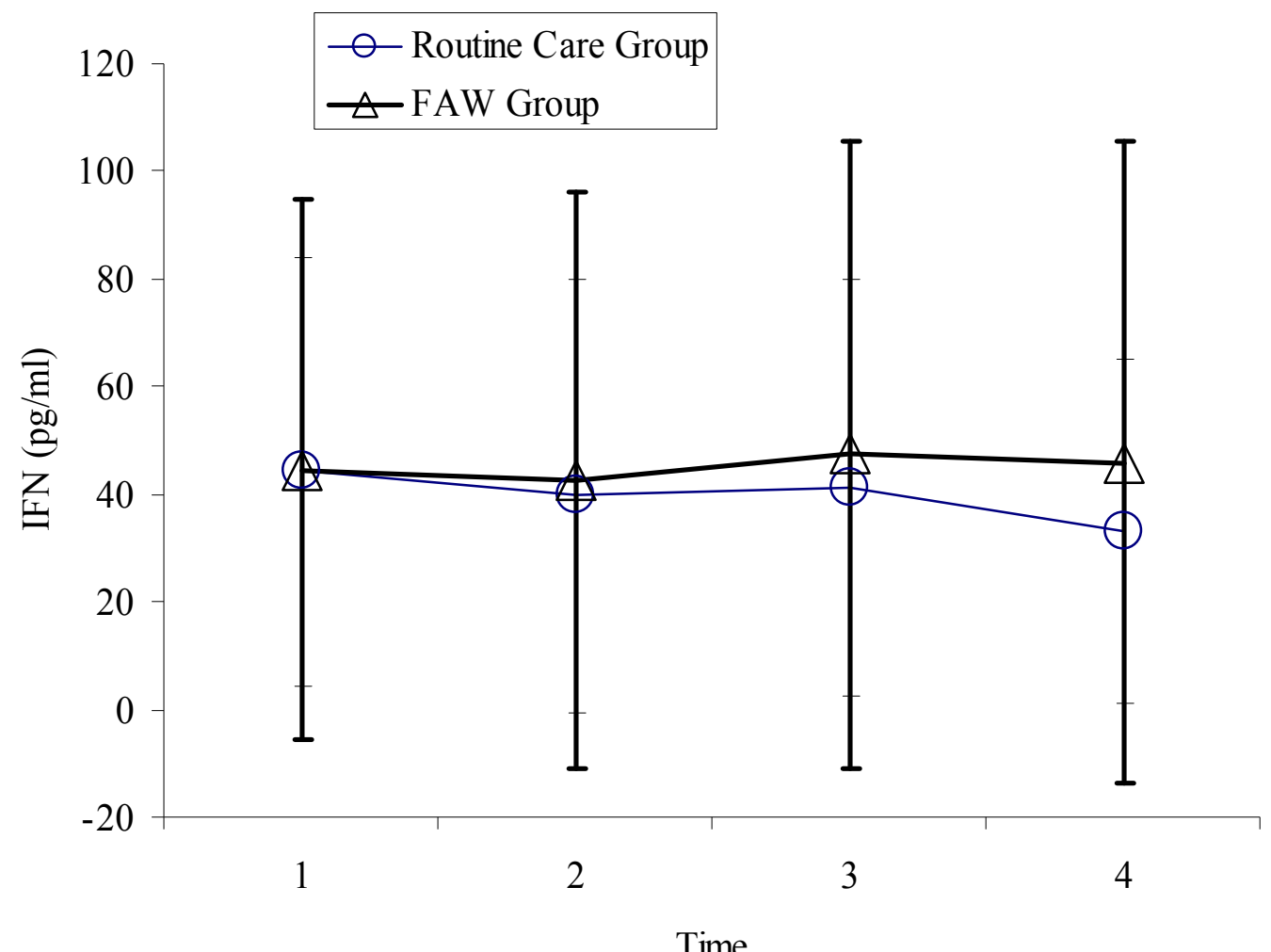

Time

$C R P$. For the measurement of CRP, there were no statistically significant main effects $(F(3,75)=1.852, p=.145)$, between subjects effects $(F(1,25)=2.190, p=$ $.151)$, or interaction effects $(F(3,75)=1.645, p=.186)$. Table 12 and Figure 11 depict the total means of CRP measurements by group over time. The statistical analysis was not supportive of the hypothesis.

Of clinical interest, there were significant findings related to CRP correlations with BMI across time. Table 13 shows these correlation coefficients. 
Table 12: Raw Mean Values of CRP (pg/ml) Across Time

\begin{tabular}{llcc}
\hline Time & Group Assignment & Mean & $\begin{array}{c}\text { Std. } \\
\text { Deviation }\end{array}$ \\
\hline CRP Time 1 & Routine Care group $(\mathrm{n}=13)$ & 6.22 & 7.20 \\
& FAW group $(\mathrm{n}=14)$ & 13.67 & 22.79 \\
CRP Time 2 & Routine Care group $(\mathrm{n}=13)$ & 3.33 & 5.51 \\
& FAW group $(\mathrm{n}=14)$ & 10.76 & 16.01 \\
CRP Time 3 & Routine Care group $(\mathrm{n}=13)$ & 3.43 & 5.06 \\
& FAW group $(\mathrm{n}=14)$ & 11.10 & 16.07 \\
CRP Time 4 & Routine Care group $(\mathrm{n}=13)$ & 3.42 & 5.05 \\
& FAW group $(\mathrm{n}=14)$ & 12.58 & 17.11 \\
\hline
\end{tabular}

Figure 11: Raw Mean Values of CRP (pg/ml) Across Time

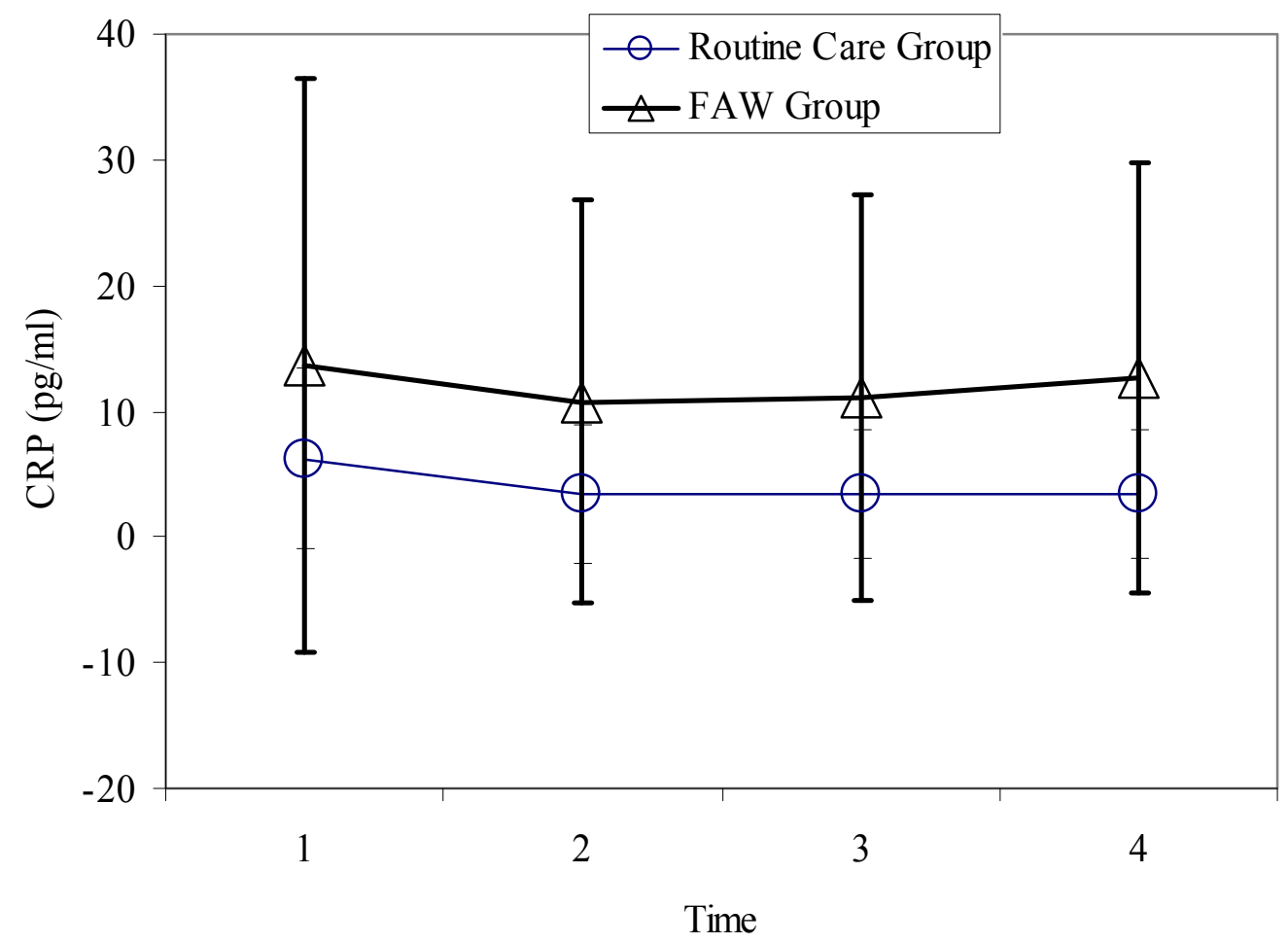


Table 13: Correlations between BMI and CRP Across Time

\begin{tabular}{llrrrr}
\hline & $\mathrm{N}=27$ & CRPT1 & CRPT2 & \multicolumn{1}{c}{ CRPT3 } & \multicolumn{1}{c}{ CRPT4 } \\
\hline BMI & Pearson Correlation & $.706\left(^{* *}\right)$ & $.693(* *)$ & $.709\left(^{* *}\right)$ & $.688\left(^{* *}\right)$ \\
& Sig. (2-tailed) & .000 & .000 & .000 & .000 \\
\hline
\end{tabular}

Note: $* *$ Correlation is significant at the 0.01 level (2-tailed).

\section{Summary}

The analytical results of this preliminary investigation into the effect of a FAW intervention on the acute phase response of surgical stress were presented. Data preparation, a profile of study participant characteristics, and statistical analyses procedures were provided. There were no statistically significant findings between the two groups of participants; therefore the proposed hypotheses were not supported. There were significant findings within groups across time with the temperature measurements, catecholamine activity, IL-6, and TNF- $\alpha$. Positive correlations between BMI and each time point for CRP were found statistically significant. The next and final chapter provides the discussion portion of these findings. 


\section{Chapter Five: Discussion}

The phenomenon of unplanned perioperative hypothermia exposes surgical patients to additional surgical stress. This has been determined by the large amount of evidence that shows the adverse perioperative effects on body systems and functions that occur in hypothermic patients (Beilin et al., 1998; Frank et al., 1997; Holtzclaw, 1997; Kurz, Sessler, \& Lenhardt, 1996; Leslie \& Sessler, 2003; Wagner, Byrne, \& Kolcaba, 2006). The more at risk the patient and the more significant the level of hypothermia, the

greater the likelihood of adverse outcomes (AORN, 2007). However, what is the effect of surgical stress of unplanned hypothermia in patients that are relatively healthy and have elected to have a routine surgical procedure? There are approximately 70 million surgical procedures performed every year in the United States and each patient may be subjected to an unwarranted surgical stress if unplanned hypothermia is allowed to occur (CDC, 1998)

This chapter provides discussion on the findings from a preliminary experimental study about the effect of a prewarming intervention on the acute phase response of surgical stress. Concluding comments about implications for both nursing and future nursing research follows a discussion regarding study limitations and lessons learned as well as how this study has contributed to nursing and nursing science.

\section{Specific Aim of the Study}

The specific aim of this preliminary study was to assess the effect of a preoperative warming intervention using a FAW device versus routine care using 
warmed cotton blankets on the development of unplanned perioperative hypothermia proinflammatory cytokine production, and neuroendocrine responses. The following hypotheses were tested and analyzed:

1. The FAW group would experience less unplanned perioperative hypothermia than the $\mathrm{RC}$ group.

2. The FAW group would experience lower catecholamine and cortisol levels than the $\mathrm{RC}$ group.

3. The FAW group would experience higher pro-inflammatory cytokine and CRP production intra and post-operatively than the $\mathrm{RC}$ group.

Each of the hypotheses will be discussed relative to the main findings.

\section{Hypothesis 1}

Hypothesis 1 predicted that the group of participants receiving FAW would experience less unplanned perioperative hypothermia than the group of patients receiving routine care with warmed cotton blankets. A review of specific temperatures by groups at Time 3 (intraoperative time frame) was conducted and there were 9 patients that had hypothermic temperatures at or below $96.8^{\circ} \mathrm{F}\left(36^{\circ} \mathrm{C}\right)$. Five of those $9(36 \%)$ were in the FAW group with a mean group temperature of $96.3^{\circ} \mathrm{F}$. The RC group $(29 \%, \mathrm{n}=4)$ were found hypothermic at Time 3 with mean temperature of $95.8^{\circ} \mathrm{F}$. Clinically, this was observed as important, as the FAW group of participants were less hypothermic $\left(96.3^{\circ} \mathrm{F}\right)$ than the RC participants $\left(95.8^{\circ} \mathrm{F}\right)$ at Time 3 by $.5^{\circ} \mathrm{F}$. A difference of .5 degree is of clinical importance. Table 13 displays the total mean temperatures at time 3 for both groups of participants that were hypothermic. 
Table 14: Total Mean Temperatures $\left({ }^{\circ} \mathrm{F}\right)$ of Hypothermic Participants at Time 3

\begin{tabular}{llcc}
\hline Time & Group Assignment & Mean ${ }^{\circ} \mathrm{F}$ & $\begin{array}{c}\text { Std. } \\
\text { Deviation }\end{array}$ \\
\hline Time 3 Temperature & RC Group $(\mathrm{n}=4)$ & 95.8 & .90 \\
& FAW Group $(\mathrm{n}=5)$ & 96.3 & .42 \\
& Total & 96.1 & .68 \\
\hline
\end{tabular}

The PACU temperatures at Time 4 of the FAW group reflected faster recovery from the hypothermic state than the RC group. Upon admission to PACU, the FAW group only had 3 hypothermic patients with an average temperature of $96.7^{\circ} \mathrm{F}$ whereas the RC group had 6 patients that were hypothermic with an average temperature of $96^{\circ} \mathrm{F}$. Table 14 shows the total mean temperatures of the hypothermic participants at Time 4.

Table 15: Total Mean Temperatures ( $\left.{ }^{\circ} \mathrm{F}\right)$ of Hypothermic Participants at Time 4

\begin{tabular}{llcc}
\hline Time & Group Assignment & Mean ${ }^{\circ} \mathrm{F}$ & $\begin{array}{c}\text { Std. } \\
\text { Deviation }\end{array}$ \\
\hline Time 4 Temperature & RC Group $(\mathrm{n}=6)$ & 96.0 & .56 \\
& FAW Group $(\mathrm{n}=3)$ & 96.7 & .10 \\
& Total & 96.4 & .57 \\
\hline
\end{tabular}

The repeated measures ANOVA statistical test and separate t-tests showed no statistical significance between groups, however, the particulars of the findings were considered clinically important. A clinical pattern of response related to temperature was also recognized in that FAW appeared to lessen perioperative hypothermia in comparison to routine care. 
The reason that the FAW group of participants became hypothermic along with the control group may be due to a preoperative impairment of thermoregulation related to the patients' premedication with midazolam (Kurz et al., 1995; Matsukawa et al., 1997). An imbalance between heat production and skin heat loss was not a large contributory predictor of hypothermia after midazolam administration in the Kurz study, however, midazolam produced a concentration-dependent decrease in core temperature $\left(.3\right.$ to $\left..6^{\circ} \mathrm{C}\right)$ and impaired thermoregulatory vasoconstriction with hypothermic redistribution occurring 30 minutes after injection (Matsukawa et al., 1997). All but one participant in the study received midazolam and there was not a significant correlation between midazolam and the hypothermic participants.

Another factor to take into consideration is the level of ambient temperature in the OR. The average Time 3 ambient temperature was $65.2^{\circ} \mathrm{F} \pm 3.6^{\circ} \mathrm{F}$. Ambient temperature is a well known predictor of core hypothermia in the anesthetized patient that shows approximately a 50\% average frequency of hypothermia occurring in ORs with temperatures of 20 to $23^{\circ} \mathrm{C}\left(68\right.$ to $73.4^{\circ} \mathrm{F}$ ) (Frank et al., 1992). The average OR temperature during this study was $18.4^{\circ} \mathrm{C}$, which is less than the known prediction temperature of ORs from the $20-23^{\circ} \mathrm{C}$ range. However, there was not a statistically significant correlation found in this study for ambient temperature and hypothermic participants.

It is difficult to treat intraoperative hypothermia because heat applied to the skin surface requires substantial time to reach the core thermal compartment and impact the temperature gradient (Sessler, 2000). The preoperative warming approach raises mean body temperature by increasing the energy content in the peripheral thermal compartment 
of the body. The amount of time needed for prewarming is still debated in the literature and ranges from 15 minutes to 120 minutes of time (Fossum, Hays, \& Henson, 2001; Melling, Ali, Scott, \& Leaper, 2001; Sessler, 1997b; Vanni, Braz, Modolo, Amorim, \& Rodrigues, 2003). Even the 15 minutes of prewarming made a difference in peripheral skin temperature and impacted unplanned hypothermia events (Sessler, 1997b). The mean amount of preoperative warming intervention during this study was 39 minutes. According to Sessler and colleagues (1995), it is possible to reduce the time of prewarming from 2 hours to 30 minutes and up to 1 hour.

The addition of at least 30 minutes of preoperative FAW warming made a clinical difference in temperature values across time and within the group with a higher level of temperature gain recognized postoperatively when compared to the $\mathrm{RC}$ group across time and within group measures. Preoperative thermal status should be considered as an important assessment point for surgical patients and the active warming approach to increase skin temperature preoperatively should be considered as a possible intervention to reduce unplanned hypothermia occurrence. As in this study, intraoperative warming is becoming more routine. There was no statistically significant covariance between groups related to intraoperative warming $(\mathrm{F}(1,16)=2.089, \mathrm{p}=.169)$.

\section{Hypothesis 2}

The prediction that the FAW group would experience lower catecholamine and cortisol levels than the RC group was not statistically supported by any of the measurement variables. However, the raw data presented differences that warranted review. Each measurement will be discussed separately. 
Cortisol. Usual serum values range from $50 \mathrm{ng} / \mathrm{ml}$ to $230 \mathrm{ng} / \mathrm{mL}$ during morning hours with a decrease seen in the afternoon/evening hours $(30 \mathrm{ng} / \mathrm{ml}$ to $150 \mathrm{ng} / \mathrm{ml})$. This study was conducted in the early morning hours and usually participation was completed by 2:00 pm in the afternoon. So the values seen in both groups of patients at Time 1 were within the range of normal. Interestingly, the values did not vary much throughout the perioperative phases under study.

Cortisol can down-regulate immune activity, but acute stress has been shown to increase both cortisol and cytokine levels. In a study by Kunz-Ebrecht and colleagues (2003), a recent investigation of individual differences in cortisol and IL-6 responses during stress showed individual variations in neuroendocrine stress responsivity. Researchers concluded that individual responsivity to stress might have an impact on proinflammatory cytokines, and that both high and low cortisol stress responsiveness had potentially unfavorable effects (Kunz-Ebrecht, Mohamed-Ali, Feldman, Kirschbaum, \& Steptoe, 2003). There are factors such as gender differences (Kirschbaum, Klauer, Filipp, \& Hellhammer, 1995), effects of age (Ershler \& Keller, 2000; Lutgendorf et al., 1999), personality factors (Pruessner et al., 1997; Smythe, 1999)and mood (Smythe, 1999) that have been found to influence cortisol as well as cytokine responses (Kunz-Ebrecht, Mohamed-Ali, Feldman, Kirschbaum, \& Steptoe, 2003). Findings suggested that those who were cortisol responsive showed little cytokine response, while the cortisol unresponsive people possibly had autonomic changes that stimulated more cytokine response. It would be interesting to evaluate responsivity in later studies and see if there is an effect on cytokines by analysis of the cytokines with a cortisol/catecholamine ratio, as this was not conducted in the present study. 
Opiods and benzodiazepines have inhibitory effects on the release of cortisol in patients undergoing surgery (Hogevold, Lyberg, Kahler, Haug, \& Reikeras, 2000; RothIsigkeit, Dibbelt, Schmucker, \& Seyfarth, 2000). In this study, all patients, but one, received the benzodiazepine midazolam preoperatively and fentanyl, an opiod type drug, intraoperatively. These medications confounded the cortisol findings, however, there were opposite changes noted in cortisol levels intraoperatively as well as immediately postoperatively. The FAW group showed a decrease in cortisol levels at Time 3 whereas the RC group had increased cortisol levels. Interestingly, the opposite was seen in the Time 4 cortisol levels with the FAW group displaying increased cortisol levels and the $\mathrm{RC}$ group showing decreased cortisol levels. Postoperative Time 4 is when the patient may be experiencing pain as well as the other effects of inflammation. Pain was not measured during this study. Whereas the differences during Time 3 may reflect the impact of the warming intervention and the levels of hypothermia postoperatively, the Time 4 cortisol levels do not support the hypothesis from a postoperative standpoint. Impact of preoperative anxiety, pain, and individual coping differences on these results may also be of importance, but these variables were not measured.

Catecholamines. Catecholamines, norepinephrine and epinephrine help the body cope with acute stress when released. An increased production of catecholamines can be found in both hypertensive and psychotic patients as well as in patients with chronic stressors. In several older studies, catecholamines increased in healthy non-anesthetized volunteers when subjected to cold ambient temperatures between $6.5^{\circ} \mathrm{C}$ and $15^{\circ} \mathrm{C}$ (Arnett \& Watts, 1960; Lamke, Lennquist, Liljedahl, \& Wedin, 1972; Wilkerson, Raven, Bolduan, \& Horvath, 1974). As expected in individuals with an active thermoregulatory 
response system, core temperature was not significantly changed during cold exposure in these studies. However, mean skin temperature was decreased by $4^{\circ} \mathrm{C}$ during a cold exposure of $15^{\circ} \mathrm{C}$. These results suggested skin surface cooling was responsible for initiating the adrenergic response. In cold water exposure testing, norepinephrine increased twofold in the first 2 minutes of exposure and increased three to fourfold after 45 minutes of exposure. The rapid norepinephrine response preceded changes in core temperature. Rewarming effects of the same volunteers resulted in a transient decrease in the first 5 minutes followed by a dramatic decrease to baseline levels with 20 minutes of rewarming. The investigators concluded that skin temperature was strongly correlated with the catecholamine response (Johnson et al., 1977; Shum, Johnson, \& Flattery, 1969). In a more recent study that assessed the relationship between body temperature, the neuroendocrine response, and hemodynamic changes in the perioperative timeframe found that patients receiving no active warming intraoperatively experienced higher norepinephrine concentrations postoperatively (Frank et al., 1995). These studies still provide support for hypothesis 2 in this study, but a lack of skin temperature measurements during this study make this association weaker. Evidence in the aforementioned studies suggests that cortisol and catecholamine release are related to immediate threats, whereas actual immune changes are sensitive to more distant events that allows for adaptive responses. Perhaps one of the reasons that the participants in this preliminary study did not show differences between groups is that all but one participant received anxiolytic benzodiazepines during the interval between Time 1 and Time 2. Benzodiazepines have been shown to reverse stress-induced immunosuppression (Zavala, 1997). The threat surrounding preoperative preparation is an acute event that preoperative 
warming might lessen and therefore decrease the level of cortisol and catecholamine release. Issues of the lack of control over anesthesia medications, and a lack of power related to small number of participants and incomplete sets of data may have diluted possible effects from the warming intervention in this study. There was no support for hypothesis 2 in these findings, however, with controls in place, further testing of how FAW may impact catecholamines remains warranted because of the regulating effects on cytokine activity during and after surgery.

Hypothesis 3. Hypothesis 3 predicted that the participants receiving FAW would experience higher pro-inflammatory cytokine and CRP production intra and postoperatively than the RC group. There was a mixture of supportive versus nonsupportive findings related to this hypothesis.

A number of characteristics relative to surgery have been associated in the postoperative suppression of cell-mediated immunity including anesthesia agents, analgesic medications, hypothermia, tissue damage, blood loss and transfusion, pain and preoperative anxiety (Shakhar \& Ben-Eliyahu, 2003). Another feature of immunity that is disturbed by surgical stress is the cytokine network, which regulates most immune functions and serves to communicate between leukocytes as well as between the immune system and other body systems. The cytokine reaction to surgery includes an immediate surge of proinflammatory cytokines (IL-1ß, IL-6), which is connected to the development of postoperative infections(Elenkov, Papanicolaou, Wilder, \& Chrousos, 1996). This preliminary surge of proinflammatory cytokines is what this study investigated.

Of greatest interest, this preliminary study showed an overall preoperative upregulation of the proinflammatory cytokines and a significant correlation across time with 
CRP and BMI. Inflammatory markers are associated with obesity (Vozarova et al., 2001), sleep disorders (Steptoe, Hamer, \& Chida, 2007), older age (Cesari et al., 2003), and cardiac conditions. Acute psychological stress has been found to influence circulating inflammatory markers (Steptoe, Hamer, \& Chida, 2007). In laboratory studies, evidence is showing that circulating levels of CRP, IL-6, and TNF- $\alpha$ markers respond to acute psycohological stress. In a meta-analysis of recent findings on the influences of acute psychological stress on circulating inflammatory markers in humans, Steptoe and colleagues (2007) addressed which markers were sensitive to acute psychological stress, the timing of the responses, and if there were differences in response related to factors such as gender, age, health status, and the nature of the acute stressor. The markers that they specifically looked at were also of interest related to this preliminary experiment. The meta-analysis procedure was performed for CRP, IL-1 $\beta$, IL- 6 , and TNF- $\alpha$. The analysis indicated that circulating levels of IL-6 typically increased following acute stress. They reported similar findings for IL- $1 \beta$ and less vigorous effects for CRP. There were no significant effects for TNF- $\alpha$. The overall conclusions suggested that laboratoryinduced stressors moderately increased inflammatory markers. The non-significant findings were attributed to between study variability and effect sizes. Another interesting finding was related to gender differences in cytokine stress responses. There were only two studies that examined gender differences and they showed inconsistent findings. Depression, work stress, and low socioeconomic status were associated with higher levels of cytokine responses. Of interest, the meta-analysis could find no studies that investigated the effect of acute psychological stress on CRP gene expression and no evidence that there are other sources of circulating levels other than hepatocytes (Steptoe, 
Owen, Kunz-Ebrecht, \& Mohamed-Ali, 2002). Since laboratory studies differ from clinical studies, this was recognized as an area of great need for future study in order to provide insight into the role of psychoneuroimmunological processes in both wellness and disease.

In this study, the measured variables for Hypothesis 3 were upregulated at Time 1 for both the FAW and RC groups. This population was overall healthy, but overweight to obese in regards to BMI. This may have considerable impact on the inflammatory process that is seen. Another strongly suspected factor is the preoperative anxiety that is usual, was observed, but not measured. A couple of studies have also implicated the effects of intravenous catheters and the blood drawing procedures as confounding the values found in cytokines ((Haack et al., 2002; Haack et al., 2000). The findings just from Time 1 were found to be exciting and warranting further research.

After the FAW treatment, the FAW group showed more of an increase than the RC group with all variables with the exception of TNF- $\alpha$. There was a noted trend of support for Hypothesis 3 even though the statisitical tests were not significant between groups. Again, this was a preliminary study and the findings were clinically, if not statistically, of great interest and warrant further research.

\section{Study Limitations}

This study was a preliminary field experiment and had several limitations related to control. First the discovery that more expensive assays were required to determine values of the biologic measures made possibility of having a large sample impossible from a budgeting viewpoint. The cost factor also disallowed the ability to conduct interassay assessments which impacts on the reliability of the biologic assay readings. 
Secondly, the Anesthesia Providers recognized when participants had received FAW preoperatively and would ask if they were to continue the practice. Just by this questioning, it was difficult to tell if the study activities influenced the decision to intraoperatively warm the patient. Even though they were told to conform to their usual practice, the number of patients that received intraoperative warming may have been impacted by the Anesthetists' knowledge of the prewarming study.

Another limitation of control was in regards to the incomplete sets of data for the catecholamine measurements. There were equipment problems during the first assay and two plates were run to get the first nine participants' catecholamine values. The need for a replacement plate of sample led to some measurement times with no plasma sample left for assay. The catecholamine kit was new to the researcher and quite complicated as well. This lack of control over the first 9 participant samples is a major limitation to adjust for in the future.

The last of the limitations was a significant issue in regards to internal validity. The infrared tympanic thermometer was chosen to take the core temperatures for this study based more on ease of route and level of non-invasiveness. The lack of skin temperature measurements to correlate with core temperature gains or losses is also a limitation. However, the researcher was the only one using the thermometer and took the temperatures in the same manner and in the same ear at each reading to limit error.

\section{Implications for Nursing}

Attention to temperature has long been a nursing practice and one of the earliest focal points of scientific investigation by nurses. The thermometer was probably the first instrument of precision that nurses used to monitor and diagnose patients. In fact, the 
thermometer can be considered one of the tools that led to the work of nursing science (Sandelowski, 2000).

Perioperative nurses should recognize the important role they can take in the prevention, or at least the reduction, of unplanned hypothermia events by using the principles of thermoregulation and evidence regarding prewarming. This study was conducted to determine the effect of preoperative FAW compared to RC on the acute phase response of surgical stress. Preliminary findings suggested that FAW is a positive intervention for better recovery from redistribution hypothermia. One factor that should encourage research in this area is its obvious and immediate practical applicability. Prevention is more cost-effective than the treatment of complications from unplanned hypothermia.

\section{Implications for Future Research}

This preliminary study can be used to determine the feasibility of a larger psychoneuroimmunology study in the near future. Earlier data obtained in another study that assessed the impact of a prewarming intervention on subjective thermal comfort and preoperative anxiety suggested that FAW provided thermal comfort while decreasing preoperative anxiety. To address the problem of surgical stress from a psychoneuroimmunology approach would be the combining of these two studies. Lessons learned from both would only strengthen the study model.

Additional research is required to determine whether the changes reported as clinically significant in this preliminary study are predictive of the positive perioperative outcomes of decreased surgical stress. The significance of this research was based upon the development of a better understanding of surgical stress and the potential protection 
from the harm of unplanned perioperative hypothermia. The need to better assess how FAW may impact the preoperative upregulation of cytokines and if the level of preoperative anxiety is associated was an important realization during the analysis phase of the study.

A combination of this study and the previous thermal comfort study could potentially provide evidence for best practices during the entire perioperative experience. It seems appropriate to suggest multidisciplinary team research to provide more substantial evidence to support global change in perioperative clinical practice since multiple professions care for the surgical patient.

\section{Summary}

A preliminary experimental study was conducted to investigate the effect of a preoperative warming intervention using FAW compared to the RC of warmed cotton blankets on the initial acute phase response of surgical stress. The focus of this study created an opportunity to examine how the phenomenon of unplanned hypothermia might be impacted by a nursing intervention, which in turn may lead to a reduction in surgical stress.

Though FAW was not associated with a differential endocrine or inflammatory response in this small, preliminary study, further study of forced air warming as a preoperative nursing intervention is warranted. The finding of higher than expected IL-6 levels in the preoperative period suggests a potential role for anxiety, an important factor in psychoneuroimmunological pathways that could affect recovery and healing. The relationship between surgical stress, anxiety, and preoperative IL-6 deserves further study. 


\section{References}

AORN. (2007). Recommended practices for the prevention of unplanned perioperative hypothermia. AORN Journal, 85(5), 972-974, 976-984, 986-978.

Arnett, E. L., \& Watts, D. T. (1960). Catecholamine excretion in men exposed to cold. $J$ Appl Physiol, 15, 499-500.

Baxevanis, C. N., Papilas, K., Dedoussis, G. V., Pavlis, T., \& Papamichail, M. (1994). Abnormal cytokine serum levels correlate with impaired cellular immune responses after surgery. Clin Immunol Immunopathol, 71(1), 82-88.

Beilin, B., Shavit, Y., Razumovsky, J., Wolloch, Y., Zeidel, A., \& Bessler, H. (1998). Effects of mild perioperative hypothermia on cellular immune responses. Anesthesiology, 89(5), 1133-1140.

Biffl, W. L., Moore, E. E., Moore, F. A., \& Peterson, V. M. (1996). Interleukin-6 in the injured patient. Marker of injury or mediator of inflammation? Ann Surg, 224(5), 647-664.

Bjornsson, G. L., Thorsteinsson, L., Gudmundsson, K. O., Jonsson, H., Jr., Gudmundsson, S., \& Gudbjornsson, B. (2007). Inflammatory cytokines in relation to adrenal response following total hip replacement. Scand J Immunol, 65(1), 99105.

Buggy, D. J., \& Crossley, A. W. (2000). Thermoregulation, mild perioperative hypothermia and postanaesthetic shivering. Br J Anaesth, 84(5), 615-628. 
Caldwell, F. T., Jr., Wallace, B. H., \& Cone, J. B. (1994). The effect of wound management on the interaction of burn size, heat production, and rectal temperature. J Burn Care Rehabil, 15(2), 121-129.

CDC. (1998). Advanced data, from the vital and health statistics of the Centers for Disease Control and Prevention. Retrieved. from.

Cesari, M., Penninx, B. W., Newman, A. B., Kritchevsky, S. B., Nicklas, B. J., SuttonTyrrell, K., et al. (2003). Inflammatory markers and onset of cardiovascular events: results from the Health ABC study. Circulation, 108(19), 2317-2322.

Connor, E. L., \& Wren, K. R. (2000). Detrimental effects of hypothermia: a systems analysis. J Perianesth Nurs, 15(3), 151-155.

Edwards, S. (2003). Cellular pathophysiology. Part 1: Changes following tissue injury. Prof Nurse, 18(10), 562-565.

Elenkov, I. J., Iezzoni, D. G., Daly, A., Harris, A. G., \& Chrousos, G. P. (2005). Cytokine dysregulation, inflammation and well-being. Neuroimmunomodulation, 12(5), 255-269.

Elenkov, I. J., Papanicolaou, D. A., Wilder, R. L., \& Chrousos, G. P. (1996). Modulatory effects of glucocorticoids and catecholamines on human interleukin-12 and interleukin-10 production: clinical implications. Proc Assoc Am Physicians, 108(5), 374-381.

Ershler, W. B., \& Keller, E. T. (2000). Age-associated increased interleukin-6 gene expression, late-life diseases, and frailty. Annu Rev Med, 51, 245-270.

Field, A. (2005). Discovering Statistics Using SPSS (2nd Edition ed.). Thousand Oaks, CA: Sage Publications, Inc. 
Flores-Maldonado, A., Medina-Escobedo, C. E., Rios-Rodriguez, H. M., \& FernandezDominguez, R. (2001). Mild perioperative hypothermia and the risk of wound infection. Arch Med Res, 32(3), 227-231.

Forstot, R. M. (1995). The etiology and management of inadvertent perioperative hypothermia. J Clin Anesth, 7(8), 657-674.

Fossum, S., Hays, J., \& Henson, M. M. (2001). A comparison study on the effects of prewarming patients in the outpatient surgery setting. J Perianesth Nurs, 16(3), $187-194$.

Frank, S. M., Beattie, C., Christopherson, R., Norris, E. J., Perler, B. A., Williams, G. M., et al. (1993). Unintentional hypothermia is associated with postoperative myocardial ischemia. The Perioperative Ischemia Randomized Anesthesia Trial Study Group. Anesthesiology, 78(3), 468-476.

Frank, S. M., Beattie, C., Christopherson, R., Norris, E. J., Rock, P., Parker, S., et al. (1992). Epidural versus general anesthesia, ambient operating room temperature, and patient age as predictors of inadvertent hypothermia. Anesthesiology, 77(2), $252-257$

Frank, S. M., Fleisher, L. A., Breslow, M. J., Higgins, M. S., Olson, K. F., Kelly, S., et al. (1997). Perioperative maintenance of normothermia reduces the incidence of morbid cardiac events. A randomized clinical trial. Jama, 277(14), 1127-1134.

Frank, S. M., Higgins, M. S., Breslow, M. J., Fleisher, L. A., Gorman, R. B., Sitzmann, J. V., et al. (1995). The catecholamine, cortisol, and hemodynamic responses to mild perioperative hypothermia. A randomized clinical trial. Anesthesiology, 82(1), 83-93. 
Gentilello, L. M., Jurkovich, G. J., Stark, M. S., Hassantash, S. A., \& O'Keefe, G. E. (1997). Is hypothermia in the victim of major trauma protective or harmful? A randomized, prospective study. Ann Surg, 226(4), 439-447; discussion 447-439.

Guest, J. D., Vanni, S., \& Silbert, L. (2004). Mild hypothermia, blood loss and complications in elective spinal surgery. Spine J, 4(2), 130-137.

Haack, M., Kraus, T., Schuld, A., Dalal, M., Koethe, D., \& Pollmacher, T. (2002). Diurnal variations of interleukin-6 plasma levels are confounded by blood drawing procedures. Psychoneuroendocrinology, 27(8), 921-931.

Haack, M., Reichenberg, A., Kraus, T., Schuld, A., Yirmiya, R., \& Pollmacher, T. (2000). Effects of an intravenous catheter on the local production of cytokines and soluble cytokine receptors in healthy men. Cytokine, 12(6), 694-698.

Haddad, J. J., Saade, N. E., \& Safieh-Garabedian, B. (2002). Cytokines and neuroimmune-endocrine interactions: a role for the hypothalamic-pituitary-adrenal revolving axis. J Neuroimmunol, 133(1-2), 1-19.

Heier, T., Clough, D., Wright, P. M., Sharma, M. L., Sessler, D. I., \& Caldwell, J. E. (2002). The influence of mild hypothermia on the pharmacokinetics and time course of action of neostigmine in anesthetized volunteers. Anesthesiology, 97(1), 90-95.

Hogevold, H. E., Lyberg, T., Kahler, H., Haug, E., \& Reikeras, O. (2000). Changes in plasma IL-1beta, TNF-alpha and IL-6 after total hip replacement surgery in general or regional anaesthesia. Cytokine, 12(7), 1156-1159.

Holtzclaw, B. J. (1993). Monitoring body temperature. AACN Clin Issues Crit Care Nurs, $4(1), 44-55$. 
Holtzclaw, B. J. (1997). Perioperative problems: threats to thermal balance in the elderly. Semin Perioper Nurs, 6(1), 42-48.

Johnson, D. G., Hayward, J. S., Jacobs, T. P., Collis, M. L., Eckerson, J. D., \& Williams, R. H. (1977). Plasma norepinephrine responses of man in cold water. J Appl Physiol, 43(2), 216-220.

Kehlet, H., \& Wilmore, D. W. (2002). Multimodal strategies to improve surgical outcome. Am J Surg, 183(6), 630-641.

Kirschbaum, C., Klauer, T., Filipp, S. H., \& Hellhammer, D. H. (1995). Sex-specific effects of social support on cortisol and subjective responses to acute psychological stress. Psychosom Med, 57(1), 23-31.

Kunz-Ebrecht, S. R., Mohamed-Ali, V., Feldman, P. J., Kirschbaum, C., \& Steptoe, A. (2003). Cortisol responses to mild psychological stress are inversely associated with proinflammatory cytokines. Brain Behav Immun, 17(5), 373-383.

Kurz, A., Sessler, D. I., Annadata, R., Dechert, M., Christensen, R., \& Bjorksten, A. R. (1995). Midazolam minimally impairs thermoregulatory control. Anesth Analg, 81(2), 393-398.

Kurz, A., Sessler, D. I., Christensen, R., \& Dechert, M. (1995). Heat balance and distribution during the core-temperature plateau in anesthetized humans. Anesthesiology, 83(3), 491-499.

Kurz, A., Sessler, D. I., \& Lenhardt, R. (1996). Perioperative normothermia to reduce the incidence of surgical-wound infection and shorten hospitalization. Study of Wound Infection and Temperature Group. N Engl J Med, 334(19), 1209-1215. 
Lamke, L. O., Lennquist, S., Liljedahl, S. O., \& Wedin, B. (1972). The influence of cold stress on catecholamine excretion and oxygen uptake of normal persons. Scand $J$ Clin Lab Invest, 30(1), 57-62.

Lee, S. L., Battistella, F. D., \& Go, K. (2001). Hypothermia induces T-cell production of immunosuppressive cytokines. J Surg Res, 100(2), 150-153.

Lenhardt, R., Marker, E., Goll, V., Tschernich, H., Kurz, A., Sessler, D. I., et al. (1997). Mild intraoperative hypothermia prolongs postanesthetic recovery. Anesthesiology, 87(6), 1318-1323.

Leslie, K., \& Sessler, D. I. (2003). Perioperative hypothermia in the high-risk surgical patient. Best Pract Res Clin Anaesthesiol, 17(4), 485-498.

Lopez, M., Sessler, D. I., Walter, K., Emerick, T., \& Ozaki, M. (1994). Rate and gender dependence of the sweating, vasoconstriction, and shivering thresholds in humans. Anesthesiology, 80(4), 780-788.

Lutgendorf, S. K., Garand, L., Buckwalter, K. C., Reimer, T. T., Hong, S. Y., \& Lubaroff, D. M. (1999). Life stress, mood disturbance, and elevated interleukin-6 in healthy older women. J Gerontol A Biol Sci Med Sci, 54(9), M434-439.

Macario, A., \& Dexter, F. (2002). What are the most important risk factors for a patient's developing intraoperative hypothermia? Anesth Analg, 94(1), 215-220, table of contents.

Matsukawa, T., Hanagata, K., Ozaki, M., Iwashita, H., Koshimizu, M., \& Kumazawa, T. (1997). I.m. midazolam as premedication produces a concentration-dependent decrease in core temperature in male volunteers. Br J Anaesth, 78(4), 396-399. 
Matsukawa, T., Sessler, D. I., Sessler, A. M., Schroeder, M., Ozaki, M., Kurz, A., et al. (1995). Heat flow and distribution during induction of general anesthesia. Anesthesiology, 82(3), 662-673.

Meiler, S. E. (2006). Long-term outcome after anesthesia and surgery: remarks on the biology of a newly emerging principle in perioperative care. Anesthesiol Clin, 24(2), 255-278.

Melling, A. C., Ali, B., Scott, E. M., \& Leaper, D. J. (2001). Effects of preoperative warming on the incidence of wound infection after clean surgery: a randomized controlled trial. Lancet, 358(9285), 876-880.

Neumaier, M., Metak, G., \& Scherer, M. A. (2006). C-reactive protein as a parameter of surgical trauma: CRP response after different types of surgery in 349 hip fractures. Acta Orthop, 77(5), 788-790.

Ogawa, K., Hirai, M., Katsube, T., Murayama, M., Hamaguchi, K., Shimakawa, T., et al. (2000). Suppression of cellular immunity by surgical stress. Surgery, 127(3), 329336.

Pruessner, J. C., Gaab, J., Hellhammer, D. H., Lintz, D., Schommer, N., \& Kirschbaum, C. (1997). Increasing correlations between personality traits and cortisol stress responses obtained by data aggregation. Psychoneuroendocrinology, 22(8), 615625.

Roth-Isigkeit, A., Dibbelt, L., Schmucker, P., \& Seyfarth, M. (2000). The immuneendocrine interaction varies with the duration of the inflammatory process in cardiac surgery patients. J Neuroendocrinol, 12(6), 546-552. 
Rutherford, E. J., Fusco, M. A., Nunn, C. R., Bass, J. G., Eddy, V. A., \& Morris, J. A., Jr. (1998). Hypothermia in critically ill trauma patients. Injury, 29(8), 605-608.

Ruzic, B., Tomaskovic, I., Trnski, D., Kraus, O., Bekavac-Beslin, M., \& Vrkic, N. (2005). Systemic stress responses in patients undergoing surgery for benign prostatic hyperplasia. BJU Int, 95(1), 77-80.

Sandelowski, M. (2000). Devices \& Desires: Gender, Technology and American Nursing. North Carolina: University of North Carolina Press.

Schein, M., Rucinski, J., \& Wise, L. (1996). Perioperative normothermia and surgicalwound infection. N Engl J Med, 335(10), 748-749; author reply 749-750.

Schmied, H., Kurz, A., Sessler, D. I., Kozek, S., \& Reiter, A. (1996). Mild hypothermia increases blood loss and transfusion requirements during total hip arthroplasty. Lancet, 347(8997), 289-292.

Sessler, D. I. (1993). Perianesthetic thermoregulation and heat balance in humans. Faseb $J, 7(8), 638-644$.

Sessler, D. I. (1997a). Mild perioperative hypothermia. N Engl J Med, 336(24), 17301737.

Sessler, D. I. (1997b). Perioperative thermoregulation and heat balance. Ann N Y Acad Sci, 813, 757-777.

Sessler, D. I. (2000). Perioperative heat balance. Anesthesiology, 92(2), 578-596.

Sessler, D. I., \& Akca, O. (2002). Nonpharmacological prevention of surgical wound infections. Clin Infect Dis, 35(11), 1397-1404. 
Shakhar, G., \& Ben-Eliyahu, S. (2003). Potential prophylactic measures against postoperative immunosuppression: could they reduce recurrence rates in oncological patients? Ann Surg Oncol, 10(8), 972-992.

Shum, A., Johnson, G. E., \& Flattery, K. V. (1969). Influence of ambient temperature on excretion of catecholamines and metabolites. Am J Physiol, 216(5), 1164-1169.

Silva, J. E. (2005). Thyroid hormone and the energetic cost of keeping body temperature. Biosci Rep, 25(3-4), 129-148.

Smythe, J., Ockenfels, M.C., Porter, L., Kirschbaum, C., Hellhammer, D.H., Stone, A.A. . (1999). Stressors and mood measured on a momentary basis are associated with salivary cortisol secretion. . Psychoneuroendocrinology, 23, 353-370.

Steptoe, A., Hamer, M., \& Chida, Y. (2007). The effects of acute psychological stress on circulating inflammatory factors in humans: a review and meta-analysis. Brain Behav Immun, 21(7), 901-912.

Steptoe, A., Owen, N., Kunz-Ebrecht, S., \& Mohamed-Ali, V. (2002). Inflammatory cytokines, socioeconomic status, and acute stress responsivity. Brain Behav Immun, 16(6), 774-784.

Tander, B., Baris, S., Karakaya, D., Ariturk, E., Rizalar, R., \& Bernay, F. (2005). Risk factors influencing inadvertent hypothermia in infants and neonates during anesthesia. Paediatr Anaesth, 15(7), 574-579.

Vanni, S. M., Braz, J. R., Modolo, N. S., Amorim, R. B., \& Rodrigues, G. R., Jr. (2003). Preoperative combined with intraoperative skin-surface warming avoids hypothermia caused by general anesthesia and surgery. J Clin Anesth, 15(2), 119125. 
Vassilieff, N., Rosencher, N., Sessler, D. I., \& Conseiller, C. (1995). Shivering threshold during spinal anesthesia is reduced in elderly patients. Anesthesiology, 83(6), 1162-1166.

Vozarova, B., Weyer, C., Hanson, K., Tataranni, P. A., Bogardus, C., \& Pratley, R. E. (2001). Circulating interleukin-6 in relation to adiposity, insulin action, and insulin secretion. Obes Res, 9(7), 414-417.

Wagner, D., Byrne, M., \& Kolcaba, K. (2006). Effects of comfort warming on preoperative patients. $A O R N J, 84(3), 427-448$.

Wenisch, C., Narzt, E., Sessler, D. I., Parschalk, B., Lenhardt, R., Kurz, A., et al. (1996). Mild intraoperative hypothermia reduces production of reactive oxygen intermediates by polymorphonuclear leukocytes. Anesth Analg, 82(4), 810-816.

Wilkerson, J. E., Raven, P. B., Bolduan, N. W., \& Horvath, S. M. (1974). Adaptations in man's adrenal function in response to acute cold stress. J Appl Physiol, 36(2), 183-189.

Wilmore, D. W. (2002). From Cuthbertson to fast-track surgery: 70 years of progress in reducing stress in surgical patients. Ann Surg, 236(5), 643-648.

Zavala, F. (1997). Benzodiazepines, anxiety and immunity. Pharmacol Ther, 75(3), 199216. 
Appendices 


\section{Appendix A: IRB Approval}

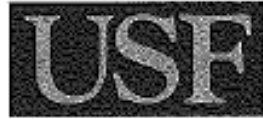

UNIVERSITY OF

SOUTH FLORIDA

March 28, 2007

Vanda Doreen Wagner, RN, MSN, CNOR

924 Chesterfield Place

Marietta, GA 30064

RE: Full Board Approval for Initial Review

IRB\#: $105439 \mathrm{~b}$

Title: Effect of a Preoperative Warming Intervention on the Acute Phase Response of Surgical Stress

Study Approval Period: $\underline{03 / 27 / 07}$ to $\underline{02 / 19 / 08}$

Dear Ms. Wagner:

On February 20, 2007, Institutional Review Board (IRB) reviewed and APPROVED the above application for the period indicated above including the following:

1. Application for Initial Review;

2. Dissertation proposal;

3. Informed Consent and Authorization Document (dated 03/2/07) entitled, "Understanding What Happens to Immunity and Stress Levels after Getting a Warming Intervention Before Surgery".

Please note, if applicable, the enclosed informed consent/assent documents are valid during the period indicated by the official, IRB-Approval stamp located on page one of the form. Valid consent must be documented on a copy of the most recently IRB-approved consent form. Make copies from the enclosed original.

Please reference the above IRB protocol number in all correspondence regarding this protocol with the IRB or the Division of Research Integrity and Compliance. In addition, we have enclosed an Institutional Review Board (IRB) Quick Reference Guide providing guidelines and resources to assist you in meeting your responsibilities in the conduction of human subjects research. Please read this guide carefully. It is your responsibility to conduct this study in accordance with IRB policies and procedures and as approved by the IRB.

\footnotetext{
Office of Research • Division of Research Integrity \& Complance InSTITUTIONAL REVIEW BOARDS, FWA No. 00001669

University of South Florida - 12901 Bruce B. Downs Blud., MDC035 - Tatnpa, FL 33612-4799

(813) $974-5638$ - Fax (813) $974-5618$
} 
Appendix A: Continued

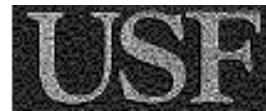

UNIVERSITY OF

SOUTH FLORIDA

We appreciate your dedication to the ethical conduct of human subject research at the University of South Florida and your continued commitment to the Human Research Protections Program. If you have any questions regarding this matter, please call 813-974-9343.

Sincerely,

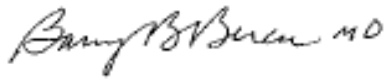

Barry B. Bercu, M.D., Chairperson

USF Institutional Review Board

Enclosures: (If applicable) IRB-Approved, Stamped Informed Consent/Assent Documents(s)

IRB Quick Reference Guide

Cc: Susan Dovhey-Phillips, PhD, USF IRB Professional Staff

Maureen Groer, PhD, RN, FAAN

IRB-Approval-FULL-0601 
Appendix B: Recruitment Flyer

\section{Interested in being WARM before your surgery?}

\section{Then you might like to be involved in an important}

\section{Nursing research study!}

\section{If so, are you: \\ Having surgery? \\ Between the ages of 18 and 65? \\ Without thyroid disease?}

The reason this research study is being done is to find out if warming a person's skin before surgery can lower stress during surgery. We are asking you to take part in the study because you are going to have surgery.

After talking with the research nurses to understand everything about the study, you will be asked to give written consent to take part in this research study.

You will only take part in the study on the day of your surgery. On the day of your surgery, you will be given either an electric warming blanket or a warmed cotton blanket while waiting for your surgery to start.

Please check the box that states your interest in this study:

$\square$ Yes, I am interested in being in this study.

$\square$ No, I am not interested at this time.

If you want to contact the nurse researcher at any time, please call Doreen Wagner, RN, MSN at 404-401-4344

This nursing research has received approval from the Institutional Review Board at the University of South Florida, Tampa and from the Douglas Hospital Nursing Research Committee of the WellStar Health System. 
Appendix C: Informed Consent Form

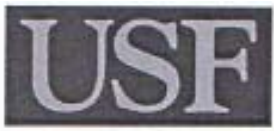

Appendix C: Informed Consent Form

UNIVERSITY OF

SOUTH FLORIDA

Informed Consent to Participate in Research and Authorization to Collect, Use, and Share Your Health Information

Information to Consider Before Taking Part in this Research Study

IRB Study \# $\underline{105439 \mathrm{~b}}$

Researchers at the University of South Florida (USF) study many topics. To do this, we need the help of people who agree to take part in a research study. This form tells you about this research study.

We are asking you to take part in a research study that is called:

Understanding What Happens to Immunity and Stress Levels after Getting a Warming Intervention Before Surgery

The person who is in charge of this research study is V. Doreen Wagner, RN, MSN, CNOR.

The research will be done at WellStar Douglas Hospital, Douglasville, Georgia.

This research is being paid for by a research grant awarded by the Association of periOperative Registered Nurses, Inc.

\section{Purpose of the Study}

The reason this research is being done is to find out if a person's internal stress that happens during surgery can be lowered by 30 minutes of skin warming with an electric warm-air blanket before surgery. Immune and hormone factors can be measured in the blood of surgical patients that will tell us if the warming experience can help lower the internal stress the body goes through during surgery.

\section{Study Procedures}

We are asking you to take part in this research study because you are going to have general anesthesia surgery and we want to find out if this warming treatment will help people who have surgery.

What will happen during this study?

We will study two groups of people.

- People in one group will have a warmed cotton blanket (feels like it just came out of the clothes dryer) given to them two times during a 30 minute time frame right before surgery. This is routine care before surgery.

- People in the other group will have an electric warm-air blanket given to them for a 30 minute time frame right before surgery. This is not usually routine care before surgery. 
Appendix C: Continued

You have a 50/50 chance of getting the routine warming treatment or the experimental warming treatment. For this study, you and the nurse researcher will not know which group you are in before you arrive for your surgery. The group you will be assigned to is randomly chosen by a computer and seen only when you come for your surgery. There will be no special treatment given to anyone taking part in the study. Everyone will have the same $50 / 50$ chance of being in one group or the other.

If you are in the group that gets the routine warming treatment, you will not be getting anything different for warming than the usual treatment before surgery. By volunteering, you are helping us learn more about surgical stress and how preoperative warming may help.

If you are in the group that gets the experimental warming treatment, you will be getting something different than the usual warming treatment before surgery. We are doing this study to find out if the electric warm-air blanket type of warming will help patients more than the usual way of warming with warmed cotton blankets.

Both groups in the study will have an extra IV line put in the arm to let the research nurse take 5 blood samples. These samples will be stored in a freezer at the hospital until time for the samples to be analyzed in the laboratory at the University of South Florida. The analysis of the blood samples will be done within 3 months of taking the samples from you.

\section{How Much Time Does It Take To Be In This Study?}

If you take part in this study, you will be asked to spend about a total of one (1) hour and 30 minutes in this study. This time will be broken down into 6 short visits.

The first time we meet will take about 15 minutes and will be completed before surgery. This visit happens after you give your written permission to be in this study. We will talk about your basic health history. We will need to know what type of surgery you are having and what day and time your surgery is to be carried out. That way we can be there on the day of your surgery to continue your participation in this study.

On the day of your surgery, your active part in the study really begins. The second and third visit with the nurse researcher will take about 40 minutes of your time. When you arrive to the preoperative area, you will be given a regular cotton gown and foot covers (footies). You will have an extra IV line put in your arm to let the nurse researcher take the 5 blood specimens during your surgery for this study. This extra IV line means only getting stuck once for the blood specimens instead of 5 times.

Before you receive any warming, you will have your vital signs taken and one tube of blood drawn from the IV line, and then you will be given either warm cotton blankets or an electric warm-air blanket. After 30 minutes of warming from either the cotton blanket or the electric warm-air blanket, your vital signs and another tube of blood will be drawn. This warming will be provided for 30 minutes and is the usual time you have to wait before you go into the operating room. The time you spend with the researcher will be when she puts in the extra IV line and at the beginning and end of the 30 minutes of warming.

The nurse researcher will also look in your medical chart to write down some other information important to the warming study. Your surgery will not be delayed in any way by participating in this study.

\begin{tabular}{|c|c|c|}
\hline partıcıpatıng in this study. & APPROVED & \\
\hline $\begin{array}{l}\text { IRB Number: } \frac{105439 \mathrm{~b}}{} \\
\text { IRB Adult ICF Template Version: } 2006-12-1\end{array}$ & $\begin{array}{l}\text { USF. INSIIIUTIONAL } \\
\text { REVIEWBOARD FWAODOOO1669 }\end{array}$ & IRB Consent Rev. Date: $\frac{03 / 2 / 07}{\text { Page } 2 \text { of } 9}$ \\
\hline
\end{tabular}


Appendix C: Continued

The fourth and fifth time you will be taking part in the study is when you are in the operating room and asleep under anesthesia. The nurse researcher will take your vital signs and one tube of blood at the start of your surgery and again at the end of your surgery. This will not stop your surgery in any way and will take about 10 minutes each time.

The last visit will take about 10 minutes of your time and happens when you are taken to the recovery room after your surgery is finished. The nurse researcher will take your vital signs and one last tube of blood. This extra IV line will be taken out at that time. There will be no other visits from the nurse researcher after your surgery.

\section{How many other people will take part?}

About 124 people will take part in this study.

\section{Alternatives}

You have the option to choose not to participate in this research study.

\section{Benefits}

We do not know if you will get any health benefits by taking part in this study. We do not know if the experimental warming before surgery will help surgical stress. That is why we are doing this study. This research study should help us learn more about whether preoperative warming measures will help the acute phase response of surgical stress.

By volunteering you are helping us learn more about how warming the skin before surgery may affect internal surgical stress. We will learn more about what does or does not help. What we learn may help others that have surgery.

\section{Risks or Discomfort}

If the surgery or the extra IV line make you feel stressed-out or uncomfortable, you can tell the nurse researcher and withdraw from the study at any time. If it is obvious that the research interaction causes participant distress, the researcher will discontinue this patient's participation in the study.

The procedure of placing an extra IV line to be used to take five tubes of blood at different times during your surgery may cause mild discomfort and a small amount of bruising. There is also a risk for bleeding and infection at the IV site.

The (forced-air) electric warming blanket causes a very low to no risk of burn injury when used. The blanket is disposable so each patient receives his or her own blanket. The electric warming blanket may make an occasional patient feel too warm, and if this occurs, the temperature of the warm air entering the blanket can be adjusted or turned off.

\section{If you are harmed while taking part in the study:}

If you believe you have been hurt or if you get sick because of something that is done during the study, you should call Dr. Maureen Groer (USF Faculty Advisor) at 813-974-2703

immediately. It is important for you to understand that the University of South Florida will not

\begin{tabular}{l|c|c}
\cline { 2 - 2 } \multicolumn{1}{c|}{ APPROVED } & \\
\hline IRB Number: $\frac{105439 \mathrm{~b}}{2}$ & & IRB Consent Rev. Date: $\frac{03 / 2 / 07}{\text { Page } 3 \text { of } 9}$ \\
IRB Adult ICF Template Version: $2006-12-1$ & USF. INSTITUTIONAL &
\end{tabular}


Appendix C: Continued

pay for the cost of any care or treatment that might be necessary because you get hurt or sick while taking part in this study. That cost will be your responsibility. Also, the University of South Florida will not pay for any wages you may lose if you are harmed by this study. The University of South Florida is considered a state agency and therefore cannot usually be sued. However, if it can be shown that your study nurse or other USF employee is negligent in doing his or her job in a way that harms you during the study, you may be able to sue. The money that you might recover from the state of Florida is limited in amount.

Medical costs that result from research-related harm may not qualify as regular medical costs. The University of South Florida may not be allowed to bill your insurance company, Medicare, or Medicaid for such costs. You should ask your insurer if you have any questions about your insurer's willingness to pay under these circumstances. The costs related to your care and treatment because of something that is done during the study will be your responsibility.

You can also call the USF Self Insurance Programs (SIP) at 1-813-974-8008 if you think:

- You were harmed because you took part in this study.

- Someone from the study did something wrong that caused you harm, or did not do something they should have done.

- Ask the SIP to look into what happened.

\section{Compensation}

We will not pay you for the time you volunteer while being in this study

\section{Confidentiality}

\section{Who will see the information that you give?}

In our research, we use and share information about people and their health. We know that this information is private. Federal law protects health information.

The law lets us use and share health information for research, if you agree to let us do this. If you sign this form, it means you are letting us use and share this information for research. If you let us use and share information about you, we will protect it as required by law.

Research at WellStar Douglas Hospital is conducted jointly with the University of South Florida. By signing this form, you are permitting WellStar Douglas Hospital and the University of South Florida to use personal health information collected about you for research purposes within WellStar Douglas Hospital health care system.

Who will disclose (share), receive, and/or use your information?

To do this research, USF and the people and organizations listed below may use or share your information. They may only use and share your information:

- With the people and organizations on this list.

- With you or your personal representative.

- As allowed by law.

USF and the people and organizations listed below may be able to see information about you and may use the information to do this research:

\begin{tabular}{l|l|l}
\cline { 2 - 3 } & APPROVED & \\
\hline IRB Number: $\frac{105439 \mathrm{~b}}{}$ & & IRB Consent Rev. Date: $\frac{03 / 2 / 07}{\text { Page } 4}$ of 9
\end{tabular}


Appendix C: Continued

- The research team, including the Principal Investigator (PI), research nurses, and all other research staff.

- All health care and other USF staff who treat and serve you as a part of this research.

- Any laboratories or other individuals and organizations that use your health information as part of the approved plan for this study.

- The sponsors of this study: Association of periOperative Registered Nurses, Inc.

- Any agency of the federal, state, or local government that regulates this research. This includes the Georgia Department of Health, and the Department of Health and Human Services (DHHS) and the Office for Human Research Protections.

- The USF Institutional Review Board and its related staff who have oversight responsibilities for this study, staff in the USF Office of Research, USF Division of Research Integrity and Compliance, and other USF offices who oversee this research.

- The designated peer review committees such as the Research Review Committee at WellStar Douglas Hospital and WellStar Health System.

Who else can use and share this information?

Anyone listed above may use consultants or others to help them understand, analyze, and conduct this study. They may use and share information about you to do this research. If you have questions about who they are, you can ask us.

Once any information leaves USF, we cannot promise that others will keep it private. USF cannot stop others from using or sharing information they have about you. The sponsor may share your information. If the sponsor or others share your information, federal privacy laws may no longer protect your information.

Others not listed here may be able to get information about you from those listed above who are not required by law to keep your information private.

\section{How will my information be used?}

By signing this form, you are giving your permission to use and/or share your health information as described in this document for any and all study related purposes. For example, your information may be used as necessary for your research-related treatment, to collect payment for your research-related treatment (when applicable) and to conduct regular business operations. Your authorization (permission) to use your health information will not expire until the end of this research study unless you revoke this authorization in writing.

As part of the research, USF may collect, use, and share the following information:

- Your whole research record

You may not want USF to use or share some kinds of information. You can list any of the kinds of information that you do not want us to use or share in the space below. If you list nothing here, we can use and share all of the information listed above. If any of the information that you do not wish us to use or share is necessary for USF to conduct the research, then you may not be able to participate in this research study.

For the Research Participant to complete:

I am asking you not to include, use, or share the following information (if blank, then no

IRB Number: $105439 \mathrm{~b}$

IRB Adult ICF Template Version: 2006-12-1

\begin{tabular}{|c|c|}
\hline APPROVED & \\
\hline ; $\quad \cdots$ & IR $\beta$ Consent Rev. Date: $\frac{03 / 2 / 07}{\text { Page } 5 \text { of } 9}$ \\
\hline
\end{tabular}


information will be excluded):

We may publish what we learn from this study. If we do, we will not let anyone know your name. We will not publish anything else that would let people know who you are.

\section{Your Rights:}

You can refuse to sign this form. If you do not sign this form:

- You will not be able to take part in this research.

- This will not change your health care outside of this study.

- This will not change your health care benefits.

- This will not change the costs of your health care.

\section{How Do I Withdraw Permission to Use My Information?}

You can revoke (withdraw) this form at any time by sending a signed letter to USF at the address given below, stating that you wish to withdraw your consent to participate in the research and to the use of your information in the research. If you revoke this form, it will tell USF that you do not want to continue to participate in the research and that you do not want USF to use or share your information anymore. If you revoke this form:

- You will no longer be a participant in this research study.

- We will stop collecting new information about you.

- The information that we have collected before you tell us to stop may already have been used or shared, or we may need it to complete and protect the validity of the research so you cannot withdraw your consent to let us use that information.

To revoke this form, you must tell us in writing. Please write to:

Principal Investigator

For IRB Study \# $105439 \mathrm{~b}$

924 Chesterfield Place

Marietta, GA 30064

While we are doing this research, we cannot let you see or copy the research information we have about you. After the research is done, you have a right to see and copy the information about you, as allowed by USF policies.

\section{How will you keep my information confidential?}

There are federal laws that say we must keep your study records private. We will keep the records of this study private by using a code number instead of your name. All data will be kept locked in a file cabinet in the Pl's office. All computer files will require passcodes.

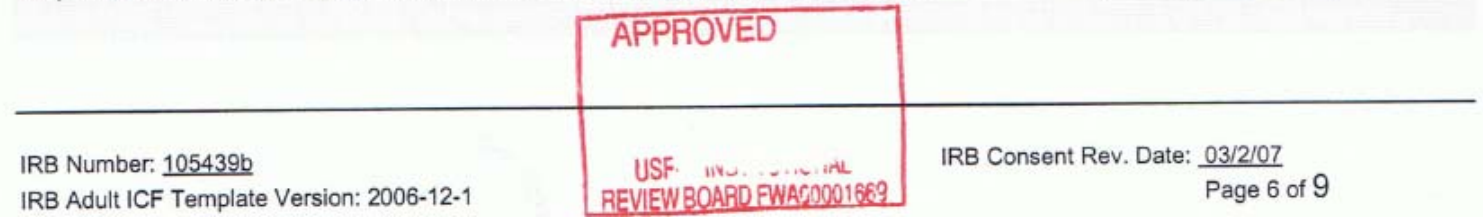


Appendix C: Continued

We will keep the records of this study confidential by assigning a code number. Consent forms will be kept separate from data. Consents and data will be stored in a locked file cabinet for 5 years in the Pl's office. Data will be transcribed into computer files that will require passcodes. Only the $\mathrm{PI}$ and the Faculty Advisor will have access to the data. Computer files will be placed on a CD for storage. Shredding of the paper files and the CD will be completed at the end of 5 years.

However, certain people may need to see your study records. By law, anyone who looks at your records must keep them completely confidential. The only people who will be allowed to see these records are:

- Certain government and university people who need to know more about the study. For example, individuals who provide oversight on this study may need to look at your records. These include the University of South Florida Institutional Review Board (IRB) and the staff that work for the IRB. Individuals who work for USF that provide other kinds of oversight to research studies may also need to look at your records.

- Other individuals who may look at your records include: the Georgia Department of Health, people from the Department of Health and Human Services (DHHS) and from the Office for Human Research Protections (OHRP). This is done to make sure that we are doing the study in the right way. They also need to make sure that we are protecting your rights and your safety.

- People at the company who paid for this study (the Association of periOperative Registered Nurses, Inc.) may look at the study records and pertinent portions of your medical records to make sure the study is done in the right way.

\section{Voluntary Participation/Withdrawal}

You should only take part in this study if you want to volunteer. You should not feel that there is any pressure to take part in the study, to please the investigator or the research staff. You are free to participate in this research or withdraw at any time. There will be no penalty or loss of benefits you are entitled to receive if you stop taking part in this study.

\section{Questions, concerns, or complaints}

If you have any questions, concerns or complaints about this study, call V. Doreen Wagner, RN, MSN, CNOR at (770) 919-9407.

If you have questions about your rights, general questions, complaints, or issues as a person taking part in this study, call the Division of Research Integrity and Compliance of the University of South Florida at (813) 974-9343.

If you experience an adverse event or unanticipated problem call V. Doreen Wagner, RN, MSN, CNOR at (770) 919-9407.

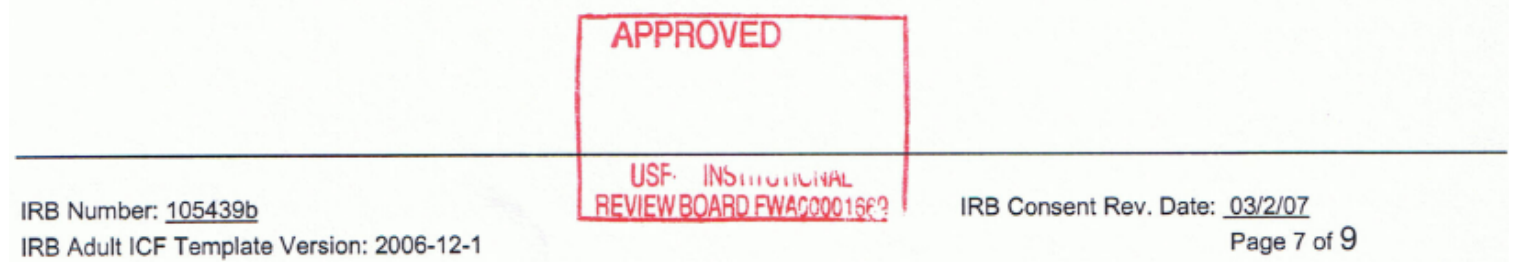




\section{Appendix C: Continued \\ Statement Of Participation in Research and Authorization for the Collection, Use and Disclosure of Health Information}

It is up to you to decide whether you want to take part in this study. If you want to take part, please sign the form, if the following statements are true.

I freely give my consent to take part in this study and authorize that my health information as agreed above, be collected/disclosed in this study. I understand that by signing this form I am agreeing to take part in research. I have received a copy of this form to take with me.

Signature of Person Taking Part in Study

Date

Printed Name of Person Taking Part in Study

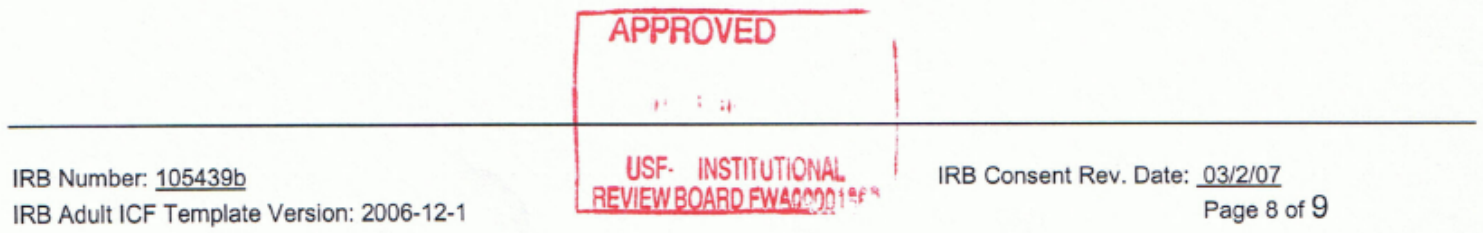


Appendix C: Continued

\section{Statement of Person Obtaining Informed Consent/Research Authorization}

I have carefully explained to the person taking part in the study what he or she can expect.

I hereby certify that when this person signs this form, to the best of my knowledge, he or she understands:

- What the study is about.

- What procedures/interventions/investigational drugs or devices will be used.

- What the potential benefits might be.

- What the known risks might be.

I also certify that he or she does not have any problems that could make it hard to understand what it means to take part in this research. This person speaks the language that was used to explain this research.

This person reads well enough to understand this form or, if not, this person is able to hear and understand when the form is read to him or her.

This person does not have a medical/psychological problem that would compromise comprehension and therefore makes it hard to understand what is being explained and can, therefore, give informed consent.

This person is not taking drugs that may cloud their judgment or make it hard to understand what is being explained and can, therefore, give informed consent.

Signature of Person Obtaining Informed Consent Research Authorization

Date

$\overline{\text { Printed Name of Person Obtaining Informed Consent Research Authorization }}$

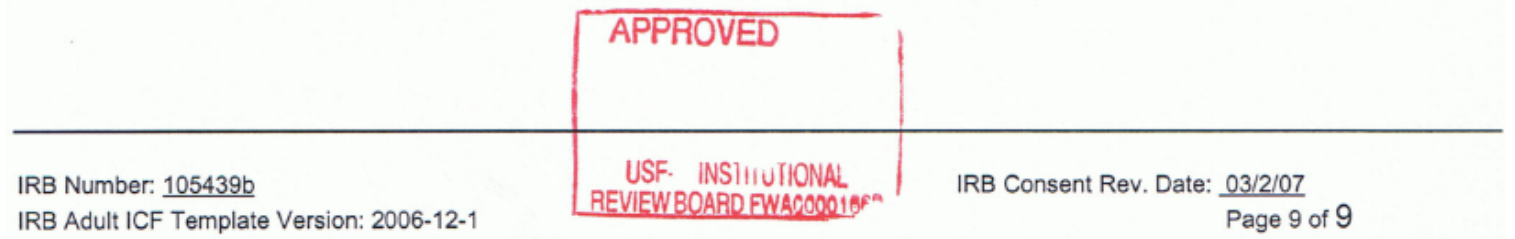




\section{Appendix D: Surgeon Letter}

April 12, 2007

WellStar Douglas Hospital Surgeon:

It is my pleasure to inform you about a nursing study soon to be implemented at Wellstar Douglas Hospital. This research is a dissertation study funded by a grant from the Association of periOperative Registered Nurses, Inc.

The purpose of this experimental study is to determine the effect of preoperative warming with forced-air warming blankets versus warmed cotton bath blankets on the acute phase response of surgical stress. All outpatient surgical patients will be recruited with "Interested" flyers.

Inclusion criteria for subjects in this study are:

Between 18 and 65 years of age

English speaking

Temperature WNL

P1 or P2 ASA physical status classification

No known cognitive impairments

No paralysis or known paresthesia

No known hypo/hyperthyroid disease

General anesthesia surgery scheduled for $>1$ hour and $<3$ hours (no scope cases)

Clean/clean-contaminated procedures only

Approval was received from both the WellStar Medical Devices Review Committee and the Institutional Review Board of the University of South Florida,Tampa. This nursing study is to begin approximately on April 19, 2007 with data collection completed by the end of June. Study results will be offered in a manuscript form for WellStar publications.

The perioperative registered nurse researcher conducting this dissertation study is Doreen Wagner, RN, MSN, CNOR (principal investigator). A full proposal of this study is available per request. Please feel free to contact me for any concerns or issues related to the inclusion of your patients in this study.

Respectfully,

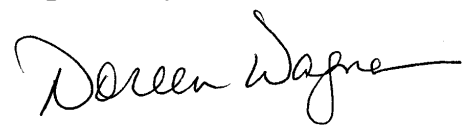

Doreen Wagner, RN, MSN, CNOR

924 Chesterfield Place

Marietta, Georgia 30064

770-919-9407/404-401-4344

doreen1799@aol.com 


\section{Appendix E: Anesthesia Provider Letter}

April 12, 2007

Dear Anesthesia Providers:

It is my pleasure to inform you about a nursing study soon to be implemented at Wellstar Douglas Hospital. This research is a dissertation study funded by a grant from the Association of periOperative Registered Nurses, Inc.

The purpose of this experimental study is to determine the effect of preoperative warming with forced-air warming blankets versus warmed cotton bath blankets on the acute phase response of surgical stress. All outpatient surgical patients will be recruited with "Interested" flyers. Inclusion criteria for subjects in this study are:

Between 18 and 65 years of age

English speaking

Temperature WNL

P1 or P2 ASA physical status classification

No known cognitive impairments

No paralysis or known paresthesia

No known hypo/hyperthyroid disease

General anesthesia surgery scheduled for $>1$ hour and $<3$ hours (no scope cases)

Clean/clean-contaminated procedures only

Approval was received from both the WellStar Medical Devices Review Committee and the Institutional Review Board of the University of South Florida,Tampa. This nursing study is to begin approximately on April 19, 2007 with data collection completed by the end of June. Study results will be offered in a manuscript form for WellStar publications.

The perioperative registered nurse researcher conducting this dissertation study is Doreen Wagner, RN, MSN, CNOR (principal investigator). A full proposal of this study is available per request. Please feel free to contact me for any concerns or issues related to the inclusion of your patients in this study.

Respectfully,<smiles>CCOC(=O)NC(C)C</smiles>

Doreen Wagner, RN, MSN, CNOR

924 Chesterfield Place

Marietta, Georgia 30064

770-919-9407/404-401-4344

doreen1799@aol.com 


\section{Appendix F: Data Collection Forms}

Name Date

Code number

\section{Does the Subject meet the following inclusion criteria?}

$\geq 18$ to $\leq 65$ years of age

English speaking

No known hypo/hyperthyroid disease No cognitive impairment

General anesthesia surgery scheduled

Clean/clean-contaminated procedure scheduled

Duration of procedure $\geq 1 \mathrm{~h} \leq 3 \mathrm{~h}$ P1 or P2 ASA classification

No paralysis or known paresthesia

Temperature WNL

Immunosuppressives $>6$ mos ago

Radiation therapy $>6$ mos ago

Tolerates tympanic thermometry

Informed Consent signed with copy given to patient: __ yes no

\section{Interview/Chart Review:}

Preoperative Diagnosis

Surgical Procedure

Date of Surgery

Arrival Time to Preop

Time of Surgery

Surgeon Name Other

Age: Sex: male female

Weight: lbs.

Height

Race/Ethnicity:

List Chronic Illnesses:

List routine medications at home (including alcohol, antipsychotics, antidepressants, OTC sedatives, etc): 
Date

Appendix F: Continued

Time I: BEFORE INTERVENTION SURVEY
(Upon Admission to Pre-operative Area)
red with one non-warmed cotton bath blanket or cotton sheet.

- $\quad$ No activation of warming blanket nor application of warmed blanket at this time.

\section{Physical data:}

Preop Room \# Ambient temperature of preoperative room

Preop Vital Signs: Tympanic T: $\mathrm{P}$ $\mathrm{R}$ BP $\mathrm{O} 2$ sat $\%$

INT site INT cath size Blood samples drawn: Time drawn

Pre-op meds or prep (at home)

At end of assessment session, provide preoperative warming intervention:

Time warmed cotton blanket x 1 applied (control group)

Temperature of warmed cotton blanket Blanket warmer temperature

Time Bair Hugger ${ }^{\mathrm{TM}}$ system activated (treatment group)

Control settings at Bair Hugger ${ }^{\mathrm{TM}}$ system activation: _ _ off _ _ cooler _ _ medium _ _ warmer Comments: 


\section{Appendix F: Continued}

Date

Code number

\section{Time II: AFTER INTERVENTION SURVEY}

*Before surgery (at least 30 after intervention)

Completion of warming intervention - either warmed blanket(s) or FAW blanket

\section{Physical data:}

Ambient temperature of preoperative room

Preop Vital Signs: Tympanic T:

$\mathrm{P}$

$\mathrm{R}$

$\mathrm{BP}$

$\mathrm{O} 2$ sat $\%$

Blood samples drawn:

Time drawn

Other

Pre-op meds, preps, or treatments (given in preoperative room/holding area)

Type/amount IV Fluid

warmed not warmed

Time IV started Type of IV antibiotics

Versed (dose/route) Time given

Pepcid (dose/route) Time given

Other preoperative medications given:

Other observations/comments (use back of this page as necessary):

\section{Check all warming information that apply in preoperative area:}

o Warmed cotton blankets

*Temperature of blanket at end of 30 minutes

o \# of warmed blankets applied (if usual care group)

o $\quad$ Additional non-warmed blankets - \# of blankets

o Booties, socks, head covering

o Warmed IV fluids

o Bair Hugger ${ }^{\mathrm{TM}}$ blanket

o Bair Hugger ${ }^{\mathrm{TM}}$ system setting: _ _ off _ c cooler _ medium _ _ warmer

o Other 
Date

Code number

\section{Time III INTRAOPERATIVE SURVEY \\ (Assessment at 1 Hour Intraoperatively)}

\section{Duration of Surgical Procedure:}

Surgical Procedure:

Anesthesia start time:

Incision open time:

Incision closure time:

Anesthesia end time:

Vital Signs: $\mathrm{P}$ $\mathrm{R}$ BP $\mathrm{O} 2$ sat $\%$

\section{$\underline{\text { Tympanic temp }}$}

\section{$\underline{\text { OR ambient temp }}$}

\begin{tabular}{|c|c|c|c|c|}
\hline Time & $\begin{array}{c}\text { Temp @ 1 hour } \\
\text { intraop }\end{array}$ & $\begin{array}{c}\text { Blood Samples } \\
\text { Drawn }\end{array}$ & Time & $\begin{array}{c}\text { OR Temp @ } \\
\text { 1 hour intraop }\end{array}$ \\
\hline & & & & \\
\hline
\end{tabular}

Active warming in OR: No Yes Start time: End time:

If yes, describe active warming approaches:

Passive warming in OR: No N_ Yes __ Start time: ___ \# warmed blankets

If other than warmed blankets, describe passive warming approaches:

\section{Circle Anesthesia Agents Provided:}

Propofol

Succinylcholine

Nitrous Oxide

Oxygen

Other
Sevoflurane

Rocuronium
Isoflurane

Desflurane

Fentany
Lidocaine

EBL: 
Date

Appendix F: Continued

Code number

\section{Time IV: ADMISSION TO PACU SURVEY \\ Postoperative Assessment}

\section{Physical Data:}

Time of Arrival to PACU:

Ambient temperature of bay area:

Postop Vital Signs: Tympanic T:

$\mathrm{P}$

$\mathrm{R}$

BP

$\mathrm{O} 2$ sat $\%$

Blood samples drawn:

Time drawn

Other

Active warming in PACU: $\quad$ No

Yes Start time:

If yes, describe active warming approaches:

Passive warming in PACU: No

Yes

Start time:

If other than warmed blankets, describe passive warming approaches:

Shivering in PACU: (circle the appropriate classification)

$0 \quad$ no shivering

1 mild fasiculations of face or neck

2 visible tremor involving more than one muscle group

3 gross muscular activity involving entire body

Time of Discharge from PACU: 


\begin{abstract}
About the Author
V. Doreen Wagner has been a nurse for approximately 25 years with career roles including perioperative staff nursing to Surgical Services Educator, Patient Safety System Coordinator to Assistant Professor of Nursing. At present, Doreen is nursing faculty at Kennesaw State University and a multi-year honoree in Who's Who Among America's Teachers. She has membership in the Sigma Theta Tau International Nursing Honor Society and the National Dean's List. She also holds membership in the American Nurses Association, Southern Nurses Research Society, Georgia League for Nursing, with heavy volunteer activism in the Georgia Nurses Association (GNA) and in the Association of periOperative Registered Nurses (AORN). She was honored with the AORN National Award for Outstanding Perioperative Nursing Research in 2005. Doreen has presented at numerous conferences locally to internationally and published many journal articles. Her research interests include thermoregulation issues of the perioperative patient, preoperative anxiety, and psychoneuroimmunology.
\end{abstract}

